\title{
Dual interferometer for dynamic measurement of corneal topography
}

Jason D. Micali

John E. Greivenkamp 


\title{
Dual interferometer for dynamic measurement of corneal topography
}

\author{
Jason D. Micali and John E. Greivenkamp \\ University of Arizona, College of Optical Sciences, 1630 East University Boulevard, Tucson, Arizona 85721, United States
}

\begin{abstract}
The cornea is the anterior most surface of the eye and plays a critical role in vision. A thin fluid layer, the tear film, coats the outer surface of the cornea and serves to protect, nourish, and lubricate the cornea. At the same time, the tear film is responsible for creating a smooth continuous surface, where the majority of refraction takes place in the eye. A significant component of vision quality is determined by the shape of the cornea and stability of the tear film. A dual interferometer system for measuring the dynamic corneal topography is designed, built, verified, and qualified by testing on human subjects. The system consists of two coaligned simultaneous phase-shifting polarization-splitting Twyman-Green interferometers. The primary interferometer measures the surface of the tear film while the secondary interferometer tracks the absolute position of the cornea, which provides enough information to reconstruct the absolute shape of the cornea. The results are high-resolution and high-accuracy surface topography measurements of the in vivo tear film and cornea that are captured at standard camera frame rates. ๑ 2016 Society of Photo-Optical Instrumentation Engineers (SPIE) [DOI: 10.1117/1.JBO.21.8.085007]
\end{abstract}

Keywords: tear film; fluid layer; interferometry; contact lens; corneal topography.

Paper 160346R received May 27, 2016; accepted for publication Aug. 8, 2016; published online Aug. 31, 2016.

\section{Introduction}

The cornea is the foremost optical element of the human eye and plays a critical role in producing images on the retina. It is the largest avascular tissue in the human body (i.e., does not contain any blood vessels), which is necessary for it to remain optically transparent. Oxygen and nutrients are instead supplied by an external mechanism: the tear film. It is a thin, multilayer of fluid that is 4 to $10 \mu \mathrm{m}$ thick. ${ }^{1}$ The tear film has additional roles of protecting, cleaning, and lubricating the cornea.

The tear film is the first optical interface with the air, resulting in the largest change in refractive index that occurs in the eye. Combined with the underlying curvature of the cornea, the tear film is responsible for $\sim 70 \%$ of the eye's total refractive power. A significant portion of vision quality is determined by the shape of the cornea and stability of the tear film. However, the nature of the tear film does not guarantee a smooth nor stable optical surface. Blinking is required to refresh the tear film to replenish nutrients, remove foreign substances, and to form an optically smooth surface. In a normal eye, a blink initially destabilizes the tear film then rapidly smoothens itself over the surface of the cornea within a few seconds due to surface tension of the fluid. ${ }^{2}$ The tear film will begin to thin due to evaporation after $5 \mathrm{~s}$ and may begin to breakup after $15 \mathrm{~s}^{3}$ Breakup in the tear film typically begins with pits or canyons forming on the surface, which continues to grow in size until the recurrence of another blink. Irregularities in the tear film can result in decreased visual performance, ${ }^{4}$ physical discomfort, ${ }^{1}$ and uncomfortable contact lens wear. ${ }^{5}$

The shape of the cornea can be measured with an ophthalmic instrument known as a corneal topographer. Corneal topographers rely on the first surface reflection from the cornea, which is the tear film interface. Therefore, the overall topography of the

*Address all correspondence to: Jason D. Micali, E-mail: jdmicali@gmail.com cornea is determined by the base shape of the cornea and the structure of the tear film. Measuring and characterizing the tear film surface and cornea are of great interest in many fields, including basic research of the eye, medicine, disease, and vision correction. For example, keratoconus is a degenerative eye disorder that causes a structural weakening of the cornea. The later stages of keratoconus result in reduced vision quality and eventually a significant loss in vision. ${ }^{6}$ Detection generally occurs after vision quality has been significantly impaired, whereas corneal topographers can be used to provide a quantitative and repeatable method for early detection of keratoconus. ${ }^{7}$

One of the most common eye diseases is keratoconjunctivitis sicca, more commonly known as dry eye syndrome. Dry eye is a disorder of the tear film characterized by tear deficiency or excessive evaporation, resulting in discomfort and even damage to the corneal surface. ${ }^{8}$ It affects $4 \%$ to $6 \%$ of the adult population and as much as $15 \%$ in the elderly ( $>65$ years of age). ${ }^{9-11}$ However, dry eye syndrome is difficult to diagnose due to the reliance on a subject's self reporting of their symptoms and limited clinical measures. ${ }^{12}$ Corneal topographers have been demonstrated to provide a quantitative method for diagnosing dry eye. ${ }^{13-15}$

Another application of corneal topography is in the field of refractive surgery. Degraded vision quality is commonly the result of an incorrect amount of power within the eye. Vision can be corrected by corrective eyewear, contact lenses, or refractive surgery. Refractive surgery is one of the most invasive methods, which involves modifying the overall power of the eye by reshaping the cornea. Topographic maps of the cornea are used to determine the amount of reshaping that is necessary to improve vision. Improved performance may be gained by high-resolution characterization of the dynamic tear film to isolate the tear film structure from the base corneal shape. Pre- and 
postcharacterization of the cornea is also useful in understanding the healing process of the eye. ${ }^{16}$

A final example for the use of a high-resolution corneal topographer is also the motivation for the instrument described in this paper: soft contact lens metrology. The value of the contact lens market in the United States is placed at about $\$ 2.5$ billion, with 39.2 million contact lens wearers, and an international market that is estimated to be about three times larger. ${ }^{17}$ The majority of contact lens wear is vision correction or therapeutic, with $\sim 5 \%$ cosmetic (e.g., colored lenses). When worn, the contact lens sits on the cornea between a cushion of tear film and an outer layer of tear film. ${ }^{18}$ The role of the prelens tear film is nearly identical to the tear film on the bare eye. Vision quality and comfort are strongly influenced by the interaction of the tear film with the contact lens material. The ability to measure the corneal topography and dynamics of the tear film structure with high resolution allows for a more complete characterization of a contact lens in its operating environment. Quantitative feedback from corneal topographers can be used to develop and refine contact lens materials and designs that could improve contact lens comfort and vision correction.

Fluorescein inspection, ${ }^{19}$ differential interference contrast microscopy, ${ }^{20}$ thin film interferometry, ${ }^{21,22}$ confocal microscopy ${ }^{23}$ optical coherence tomography, ${ }^{24}$ and optical interferometry $^{25-28}$ are all methods that have been employed to study the tear film and underlying corneal structure. However, these methods have various limitations that range in their level of invasiveness, resolution, and ability to measure the dynamics of the tear film. Nor do any of these methods provide information on the absolute surface shape of the tear film and cornea.

The most common type of corneal topographers that are used in the ophthalmic community can be broken down into two domains based on their mode of operation: projection and reflection. Projection-based topographers project structured patterns onto the cornea and recover topography by measuring the distortions of the projected image. As the optical surfaces in the eye are transmissive, there is very little diffuse reflectance to make use of. Instead, most of these systems require that a diffusing or fluorescing agent be added to the tear film to increase the amount of diffuse reflectance of the cornea. There are two common forms of projection-based systems: raster-stereography ${ }^{29-31}$ and moiré-deflectometry. ${ }^{32,33}$ Surface height resolution for these types of systems have been demonstrated to be in the order of microns; however, the introduction of a foreign substance alters the behavior of the tear film making them undesirable to study the tear film. ${ }^{34}$ Furthermore, it could be argued that topographic resolution is limited by the fact that the introduction of a foreign substance alters the shape and behavior of the tear film structure.

Another form of projection-based topography uses slit scanning. A bright, thin slit is projected onto the surface of the cornea that produces enough specular light that can be resolved by an imaging system. The slit has to be scanned across the cornea to build up a topographic map of the cornea. This type of system has been demonstrated to have micron height accuracy on static surfaces. ${ }^{35}$ However, the duration of the scan can be as much as $30 \mathrm{~s}$, in which time eye motion and tear film dynamics can wash out surface information and limit height accuracy. This also limits the ability of the device to make dynamic measurements of the tear film structure. Spatial resolution for projection-based systems is in the order of $100 \mu \mathrm{m}$; limited by the projected image and recording system.
The more common type of topographer systems is reflectionbased, which relies on the specular reflectance from the tear film. An illuminated pattern is placed in front of the subject's eye, and a camera system records an image of the reflected illumination. Distortions in the image correlate to surface features from which the surface topography can be determined. The Placido disc is a commonly used illumination pattern, which consists of a series of concentric circles. Modern computerized Placido disc topographers place a camera at the center and behind the disc (Fig. 1). Reflection-based systems, such as the Placido disc topography, have been demonstrated to have similar spatial and height resolution as the projection-based systems. ${ }^{30,36}$ The advantage of a reflection-based system over a projection-based system is that they can noninvasively measure corneal topography and the tear film surface, in addition to making dynamic measurements.

What is presented in this paper is a next generation corneal topographer system that surpasses the capabilities of all known systems. The system is a dual interferometer system that allows for noninvasive, high-resolution measurements of the corneal topography and tear film surface to be captured at 30 frames per second (FPS). The limited spatial and height resolution of all known corneal topographers prevent them from fully resolving the tear film structure. Systems that are able to resolve the tear film structure and thickness contain little to no topographic information. However, the two surfaces are inextricably linked. The high-resolution corneal topographic measurements from the system presented in this paper provide not only topographic information about the cornea, but also tear film structure in both spatial and height domains. The dependence on the tear film also requires that measurements are passive and noninvasive. Measurements that are made at 30-FPS allow the tear film dynamics to be resolved, potentially allowing for the tear film structure to be isolated from the base corneal topography.

This paper describes an interferometric corneal topographer (ICT) system and presents results from testing with the device. Section 2 provides background and information related to the problem of interferometric measurements of the cornea and tear film. Section 3 describes the ICT system and reviews laser safety. Section 4 reviews data processing required to handle the data acquired by the ICT and how the corneal topography is recovered from measurements. Finally, Sec. 5 verifies the capabilities of the system and compares it to a commercial device. The final set of results are from human subject testing.

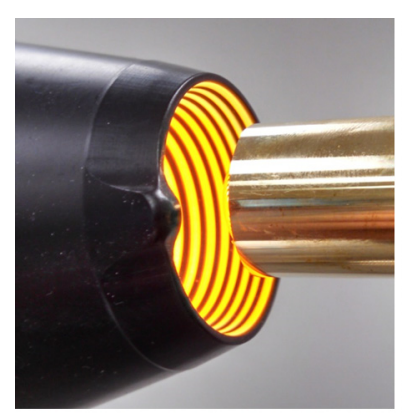

(a)

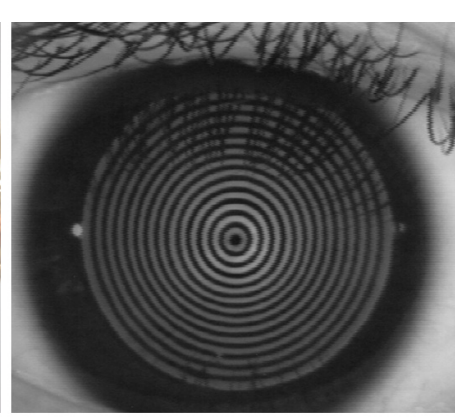

(b)
Fig. 1 Placido disc topographer. (a) Topographer measuring a simulated eye surface. (b) Topographer measuring a human eye. 


\section{Background}

\subsection{Topography of the Human Eye}

One of the challenges in the design of an interferometer to measure the topography of the human cornea is that the shape of the cornea will vary from person to person. The average corneal shape is best approximated by an asphere with a base radius of $7.8 \mathrm{~mm}$ and a -0.25 -conic constant, which is an oblate ellipsoid. ${ }^{37-39}$ The range of radii that were measured in these studies varied from 6.7 to $9.4 \mathrm{~mm}$. The distributions were normally distributed with a standard deviation of $0.3 \mathrm{~mm}$ about a mean of $7.8 \mathrm{~mm}$. The conic constant, or asphericity, was also measured in a range from -0.76 to +0.47 . The asphericity measurements were normally distributed with a standard deviation of 0.27 about the mean value of -0.25 .

A more accurate description of the cornea includes meridional variations of the base radius, which results in a toroidal shape of the cornea. The toroidal shape of the cornea is more commonly referred to as corneal astigmatism. Mean variation between meridional radii was measured to be $0.15 \mathrm{~mm}$, where the variation was defined as the difference between the minimum and maximum radii. ${ }^{37,40}$ About $\sim 74 \%$ of the study population had a larger radius close to the horizontal (i.e., within $45 \mathrm{deg})$.

Additional studies would indicate that a toroidal description of the cornea is incomplete. ${ }^{41-43}$ Surface deviation from a toroid can exceed a few microns or more due to the complex surface structure of the cornea. Tear film dynamics, especially the reforming and thinning, should be expected to add an additional range of surface variation that is comparable to the thickness of the tear film. The result from all of these effects is a complex and dynamic surface topography of the cornea that presents a significant challenge to making high-resolution interferometric measurements.

\subsection{Eye Motion}

The advantage gained by using interferometry to acquire highresolution measurements becomes a disadvantage when it comes to the increased sensitivity to misalignment. This is generally not an issue for traditional interferometry, where stable mounting and fine resolution adjustment mechanisms can be used to align and maintain the test surface relative to the interferometer. These methods could be extended to the application of interferometry on the eye; however, a noninvasive measurement of the in vivo (i.e., living) eye would not allow for an equivalent degree of securing the subject's head and/or eye. Therefore, it must be accepted that a range of random eye motion will be present during measurements that will result in a misaligned surface with respect to the interferometer.

Eye motion can be classified into two domains: voluntary and involuntary. The most basic voluntary eye motion is gaze, where a coordination of eye and head motion allows a person to fixate on and track an object. Head motion is minimized by placing the subject's head in a restraining fixture. A common fixture is comprised of a chin cup and forehead rest. One study showed that head motion in these types of fixtures was in the range of $\pm 100 \mu \mathrm{m}$ and strongly correlated to the subject's breathing and heartbeat. $^{44}$

Minimization of gaze is accomplished by directing the subject to lock their visual gaze on a stationary target. Despite the ability of a subject to fixate, the eye will make small, random, and involuntary motions. ${ }^{45,46}$ Involuntary eye motion is decomposed into three types of motion: saccades, drifts, and physiological nystagmus or tremors. Saccades are quick, large flicks of the eye that occur one to three times per second with an average magnitude of 6 arc min. ${ }^{47}$ Drifts are slow, cyclic motions that occur at a rate of a few arc $\mathrm{min} / \mathrm{s} .{ }^{48}$ Physiological nystagmus is a small, random, and high-frequency $(50$ to $100 \mathrm{~Hz})$ motion with a magnitude slightly greater than 2 min of arc. ${ }^{47}$

Previous studies have shown that color, luminance, contrast, or image quality of the fixation target have no effect on fixation except in the case where any of these factors render the target barely visible. ${ }^{49,50}$ However, it was found that differences in fixation target sizes resulted in changes in saccades and drift. ${ }^{50,51}$ External distractions, such as motion in the periphery or auditory stimuli, were also found to increase the amount of microsaccades. ${ }^{52,53}$ A noninvasive measurement of the cornea and tear film must, therefore, accept that a certain amount of motion will be present. If eye motion is left unaccounted for, it will significantly degrade or inhibit the ability of the instrument to measure the cornea and tear film.

\subsection{Soft Contact Lenses}

A soft contact lens is a thin (70 to $200 \mu \mathrm{m}$ ) hydrogel material that is placed directly on the surface of the eye. A hydrogel is a hydrophilic polymer that is highly absorbent, with the water content of a fully hydrated hydrogel lens ranging anywhere from $24 \%$ to $74 \% .{ }^{54} \mathrm{~A}$ recent development in the last decade has been to introduce silicone into the hydrogel, which increases oxygen permeability allowing for longer continuous wear. By varying water content, silicone, and other parameters of a contact lens, a manufacturer can adjust the optical characteristics, comfort, and a number of other factors.

When worn, the soft contact lens sits on a cushion of tear film that is $\sim 2-\mu \mathrm{m}$ thick. ${ }^{18}$ A prelens tear film forms on the anterior surface of the contact lens that has similar function to the tear film on the bare eye. Vision correction is an obvious requirement for the design of a contact lens; however, an equally important requirement is comfort. Stability of the tear film may influence the comfort of the contact lens. ${ }^{5,55}$ The hydrophobic property of the silicone that is added to the hydrogel lenses may contribute to an increase in tear film breakup and may reduce vision quality. ${ }^{54}$ Therefore, it is desirable to have an instrument that is capable of resolving the interaction of the tear film surface with the contact lens to better study and characterize contact lens materials.

\subsection{Research Background and History}

For a little over a decade, the focus of this research group has been on the application of interferometry to soft contact lens metrology. ${ }^{56}$ This has led to the development of a number of instruments for characterizing the optical properties a soft contact lens. One of the first systems was a Mach-Zehnder interferometer for measuring the transmitted wavefront of the contact lens. ${ }^{57}$ Another system measured the index of refraction of the hydrogel materials. ${ }^{58}$ The most recent system was designed to measure the surface profile of both sides of the contact lens. ${ }^{59}$ The combination of measurements from these three systems allows for a complete characterization of the optical properties of the contact lens.

However, these systems are limited to testing the soft contact lenses submersed in a saline solution, which is not necessarily 


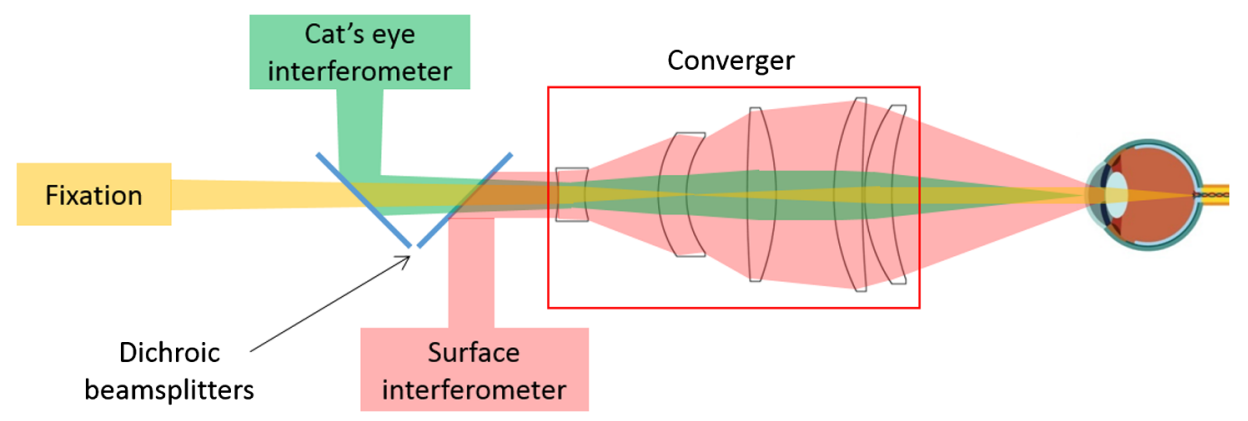

Fig. 2 ICT system.

representative of their intended use. As discussed in Sec. 2.3, the contact lens sits between layers of the tear film. Exposure to the environment, interaction with the tear film, variation in corneal topography, and external forces such as blinking, results in a dynamic environment that adds an additional layer of complexity to the design of a contact lens. The next phase in soft contact lens metrology is the ability to measure the contact lenses in vivo (i.e., measured directly on the living eye).

The development of an interferometer for measuring the in vivo tear film and corneal topography has been accomplished in three phases. The first phase demonstrated an interferometer for measuring the dynamics of a fluid layer on a surface. ${ }^{60}$ This was an in vitro measurement (i.e., outside of the body) of a simulated tear film layer on a contact lens surface placed on an artificial eye surface. This system has the advantage of being able to characterize the dynamics of a fluid layer surface on a contact lens without the need for a human subject. It also demonstrated that an interferometric measurement of the in vivo tear film was possible. This led to the second phase, the development of the tear film interferometer (TFI) for in vivo measurements of the tear film surface on the human cornea. ${ }^{61,62}$ The successful demonstration of the TFI has led to the third phase, an interferometer for measuring the dynamic corneal topography of the in vivo eye that is described in this paper.

\section{System Description}

The ICT system consists of a pair of polarization-splitting Twyman-Green interferometers and a fixation assembly. All

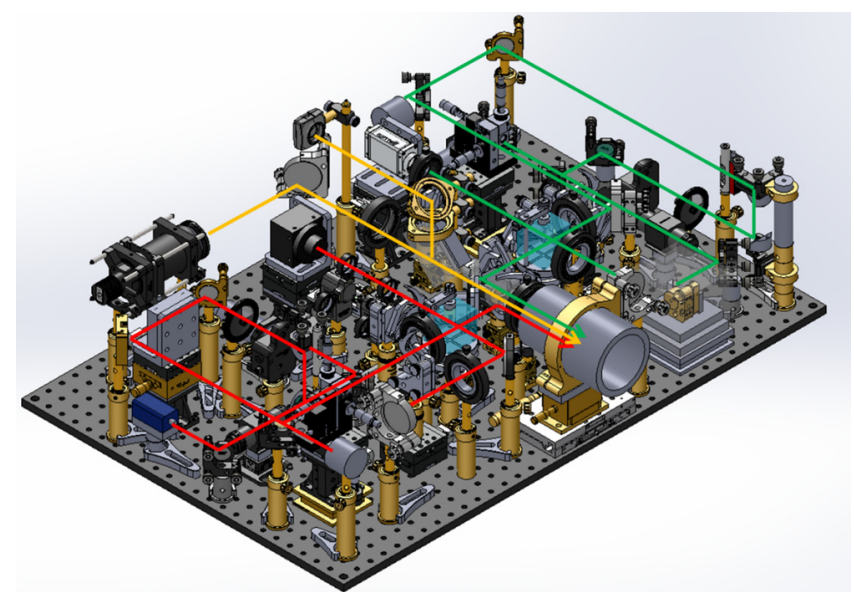

Fig. 3 Layout of the ICT system. The surface interferometer, cat's eye interferometer, and fixation assembly paths are overlaid in red, green, and yellow, respectively. three subsystems operate at different wavelengths and are coaligned at the input to a converger lens assembly using a pair of dichroic beamsplitters (Fig. 2). The converger lens has been designed as a null or compensating lens in the test path of one of the Twyman-Green interferometers to null against the average shape of the human cornea (Sec. 2.1). The converger lens covers a $6-\mathrm{mm}$ diameter on the cornea, operating at $\sim f / 1.3$. This interferometer subsystem measures the tear film surface and is therefore referred to as the surface interferometer. The surface interferometer is discussed in more detail in Sec. 3.1.

The second Twyman-Green interferometer produces a convergent wavefront that focuses $7.8 \mathrm{~mm}$ before the surface interferometer focus. When an average shaped eye (Sec. 2.1) is aligned to the surface interferometer in a confocal configuration, the secondary interferometer will be focused onto the surface of the cornea; a cat's eye configuration. Thus, the second interferometer is referred to as the cat's eye interferometer. A simultaneous measurement of any corneal surface with both systems provides enough information to reconstruct the corneal topography and tear film surface as will be discussed in Sec. 4. Details of the cat's eye interferometer are presented in Sec. 3.2.

The final subsystem is the fixation assembly, which provides a distant target for human subjects to fixate on during a test. A fixation target minimizes eye motion (Sec. 2.2), which would otherwise prevent the system from being capable of being aligned to a human subject or allowing for sequential measurements. The fixation assembly is discussed in Sec. 3.3.

A more detailed hardware layout is shown in Fig. 3, and an image of the as-built hardware is shown in Fig. 4. The optical paths for the surface interferometer, cat's eye interferometer, and fixation assembly are shown in red, green, and yellow,

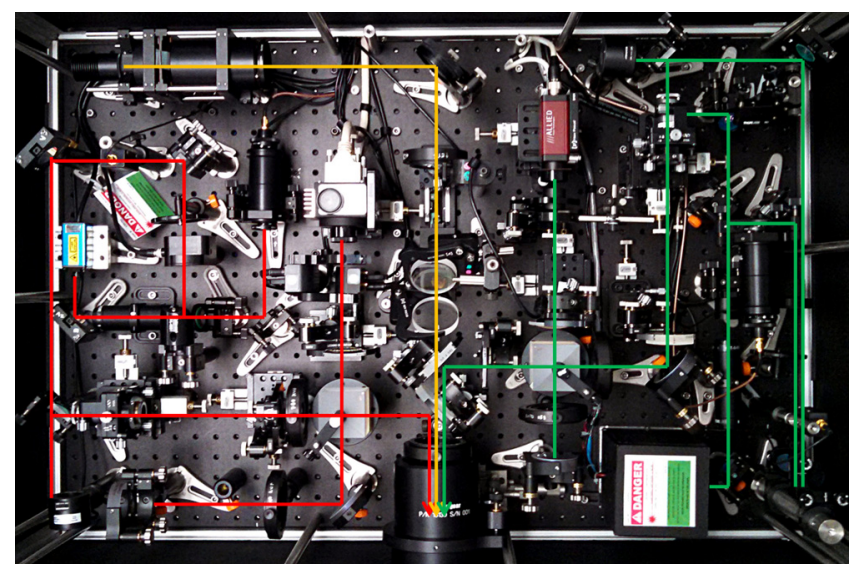

Fig. 4 Top view of the ICT system with the cover removed. 


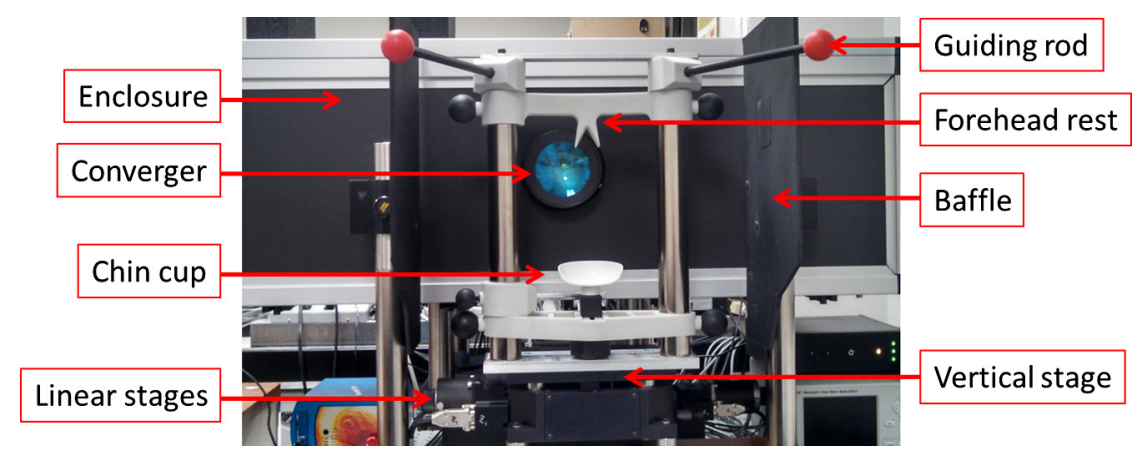

Fig. 5 External view of the ICT system.

respectively. Details of the three ray paths will be given during the discussion of each individual subsystem in the following Secs. 3.1, 3.2, and 3.3. An external view of the system is shown in Fig. 5 with key components labeled. The system is enclosed in a box to limit access to internal components and to prevent accidental exposure to the internal laser sources. The output of the system is controlled and verified to operate at eye safe levels, which is discussed further in Sec. 3.4. A subject will sit in a headrest that is placed in front of the system such that their eye is nominally aligned to the output of the converger assembly. The headrest assembly consists of a chin cup, forehead rest, and a pair of guiding rods that will wrap around the subject's head at the temples to minimize head motion. The system is set on top of motorized linear stages to allow for fine alignment of the system to the subject. Baffles are placed alongside the subject's head to minimize exposure to external distractions, which would otherwise cause an increase in eye motion (Sec. 2.2).

\subsection{Surface Interferometer}

The surface interferometer is functionally equivalent to the TFI, the predecessor to this system (Sec. 2.4). A more in-depth discussion about the TFI can be found in the Refs. 61 and 62 This section provides a brief overview of the surface interferometer, which includes modifications that have been made since the original TFI system.

The surface interferometer is a polarization-splitting Twyman-Green interferometer that acquires measurements at 30 FPS. A layout of the interferometer is shown in Fig. 6 with key elements labeled. The laser source is a near-infrared solidstate laser $(\lambda=785 \mathrm{~nm})$. The laser was found to drift over time, which resulted in a loss of coherence length and decreased fringe visibility. Instead of replacing the laser, a cost-effective solution was to pick off a small portion of the laser beam and pass it to a confocal scanning Fabry-Pérot interferometer to passively monitor the laser. The laser current diode or temperature control set-point can be adjusted to push the laser back into a stable mode. The reflected path after the pick-off beamsplitter contains a shutter and continuously variable neutral density (CVND) filter. The CVND is used to adjust the laser power to ensure an eye safe power level at the output of the interferometer.

An acousto-optical modulator (AOM) follows the CVND and is used to modulate the laser source. The AOM is synchronized to the electronic shutter of the detector (i.e., integration time). Detector integration times are limited to $100 \mu \mathrm{s}$ at frame rates of $30 \mathrm{~Hz}$, such that the modulation duty cycle is $<1 \%$. The primary purpose of the AOM is to reduce the apparent visibility of the laser source, which has been shown to distract test subjects and limit their ability to maintain fixation during testing. The AOM modulates the laser source by deflection. When the $\mathrm{AOM}$ is inactive (i.e., detector is not integrating), the nondeflected beam passes over the pick-off mirror and is incident on a power meter. The power meter is calibrated to the output of the system and is used to constantly monitor the laser power during testing to ensure that the output of the system is eye safe (Sec. 3.4).

When the AOM is active, the laser beam is deflected such that it is incident with the pick-off mirror and is reflected to an objective and spatial filter. Following the spatial filter, the diverging beam is collimated, passed through a half-wave plate, and then a polarizing beamsplitter. The beam is split between the reference and test arms of the interferometer. The test arm of the interferometer is folded to the input of the converger assembly. The fold is the first surface of a dichroic beamsplitter and is designed to reflect 785-nm light for the surface interferometer, transmit visible (400 to $700 \mathrm{~nm}$ ) for the fixation, and transmit $850 \mathrm{~nm}$ for the cat's eye interferometer. Following the dichroic is an achromatic quarter-wave plate (AQWP) that is designed to operate with the 785-nm source in the surface interferometer and the 850-nm source in the cat's eye interferometer. Fixation illumination is unaffected by the AQWP.

The converger optical assembly is designed to generate a wavefront that matches the shape of the average human cornea (Sec. 2.1). The reflected wavefront from the tear film surface is interfered with the reference wavefront and measured with a 1-MP Pixelated Camera Kit (4D Technology Corporation, Tucson, Arizona). The camera uses a pixelated phase-mask

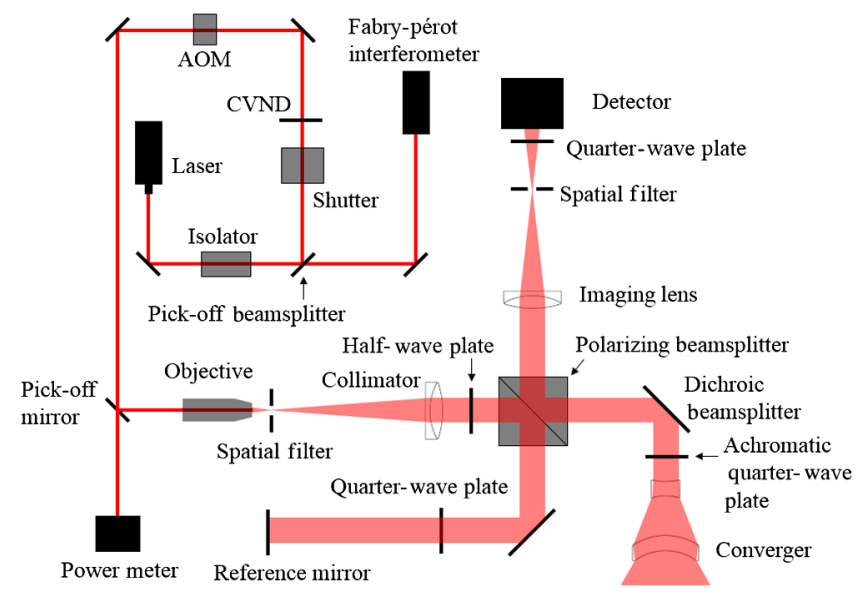

Fig. 6 Layout of the surface interferometer. 


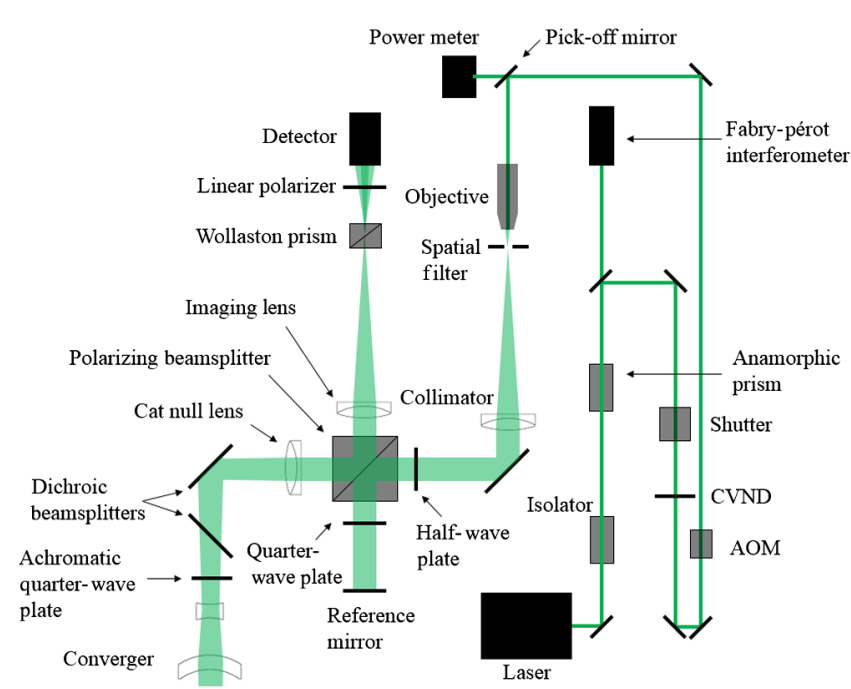

Fig. 7 Cat's eye interferometer layout.

aligned to the detector array that acquires four-phase shifted interferograms in a single camera frame. ${ }^{63}$ Phase can be recovered from an individual frame by conventional $N$-bucket algorithms or spatial convolution methods. ${ }^{63,64}$ Most of the interferograms that will be measured are dense and complicated, requiring additional postprocessing, which will be discussed in Sec. 4.1.

Surface measurements are made relative to a reference wavefront that is produced by the converger, which provides no information about the absolute surface shape of the cornea and tear film. Furthermore, most measurements will deviate from the reference wavefront due to eye motion and anatomical variation, which introduces additional interferometer errors into measurements. By itself, the surface interferometer can only recover relative height information of the tear film surface after most of the low spatial-frequency structure is backed out of the measurement. Recovery of the absolute surface topography will require measurement information from the secondary interferometer that is discussed in Sec. 3.2 section and requires additional data processing that is discussed in Sec. 4.2.

\subsection{Cat's Eye Interferometer}

The cat's eye interferometer provides a secondary measurement that allows for the absolute surface shape of the cornea and tear film surface to be recovered (Sec. 4). The absolute shape provides additional information about the cornea that cannot be determined from a surface measurement, such as the radius of curvature, asphericity, or astigmatism. A simultaneous confocal and cat's eye measurement could be accomplished by any number of methods, but to minimize the cost for this system, the cat's eye interferometer was designed as a minimally invasive add-on to the TFI system.

A hardware layout of the cat's eye interferometer is shown in Fig. 7. It is a polarization-splitting Twyman-Green interferometer that uses a spatial carrier method to measure instantaneous phase. The interferometer operates at a near-infrared wavelength $(\lambda=850 \mathrm{~nm})$. The laser source was built in-house, a cost-effective solution that is common to many experimental setups. ${ }^{65}$ The same monitoring systems that are used in the surface interferometer are implemented in the cat's eye interferometer to ensure stability (Fabry-Pérot interferometer) and safety (power meter,

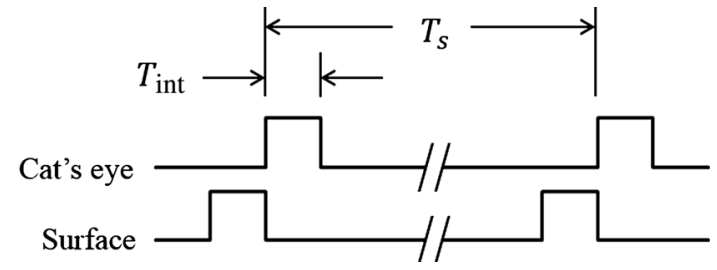

Fig. $8 \mathrm{AOM} /$ camera timing for the two interferometer systems.

CVND, shutter). The output of the laser is elliptical, which is reshaped into a circular beam with an anamorphic prism that follows a Faraday isolator. The following optical components that lead up to the polarizing beamsplitter are identical to the surface interferometer.

The test arm of the cat's eye interferometer is combined with the surface interferometer and passed through the converger optical assembly. The test path of the cat's eye interferometer is coaligned to the surface interferometer test path; misalignment between the two systems will be accounted for with the interferometer calibration (Sec. 4.3). A slightly positive meniscus lens (cat null) is placed in the test path to offset the focus of the cat's eye interferometer to the surface of the nominal cornea (i.e., a 7.8-mm radius of curvature). When a nominally shaped cornea is aligned to the surface interferometer in a confocal alignment to produce a null fringe, a null fringe is also captured with the cat's eye interferometer in a cat's eye configuration. Variation in eye shape and alignment will introduce optical path differences in the surface and cat's eye measurements that can be used to determine the absolute shape of the cornea (Sec. 4). The test arm is folded and aligned to the surface interferometer by a second dichroic beamsplitter, which is designed to reflect $850 \mathrm{~nm}$ for the cat's eye interferometer and to transmit in the visible for the fixation assembly.

The cat's eye interferometer uses a spatial carrier method to measure the phase in a single snapshot. ${ }^{66,67}$ The spatial carrier method requires a large tilt component to be introduced between the test and reference paths. This is accomplished by placing a Wollaston prism at the focus of the imaging lens. The prism is adjusted along the optical axis to control the amount of offset between the test and reference beams. An analyzer (linear polarizer) is placed between the prism and detector, and the fringes are recorded with a 4-MP detector. A large format sensor is required to resolve the dense fringes produced by the spatial carrier method, range of variation in focus due to eye motion, and anatomical variation.

There is a small amount of spectral leakage from the first dichroic beamsplitter that results in a noticeable amount of cross-talk between the two interferometer systems. To minimize cross-talk, the cat's eye camera and AOM are set to trigger off of the falling edge of the surface camera as shown in Fig. 8. The integration time $\left(T_{\text {int }}\right)$ for both systems is set to $100 \mu \mathrm{s}$, which results in a nearly simultaneous capture of the two independent measurements when compared to the overall sampling time $\left(T_{s}=33.3 \mathrm{~ms}\right)$ of the system.

\subsection{Fixation}

The purpose of a fixation target is to present a distant target for the subject to lock their gaze and minimize eye motion. Fixation is critical for operation of the ICT-eye motion is the largest contributor to misalignment of the eye with respect to the interferometer and results in nonnull measurements in the surface 
(15 $\uparrow \quad$ Target

Light source

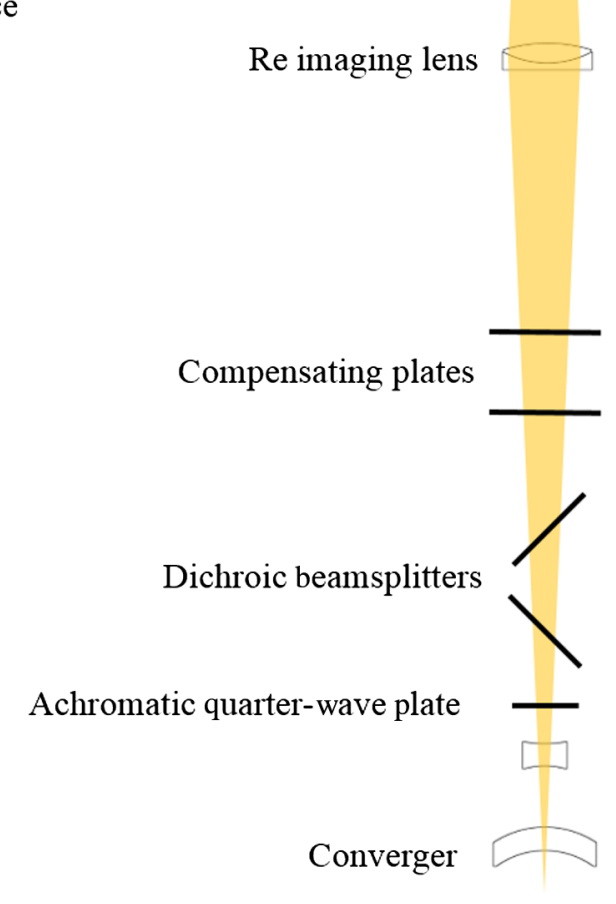

Fig. 9 Fixation assembly layout.

interferometer. The resulting fringes will exceed the capture range of the detector. Without proper fixation, the system is practically impossible to align to the subject. Baffles, stray light management, proper target choice, and target illumination are other factors that have to be considered when minimizing eye motion (Sec. 2.2).

The fixation assembly consists of a reimaging lens that images a back-illuminated target onto the back focus of the converger assembly (Fig. 9). Although it was found that the optimal fixation target is a set of cross-hairs surrounded by a circular target with a dot in the center, ${ }^{62,68}$ the target for this system is a $50-\mu \mathrm{m}$ pinhole. The features of the cross-hair target that was used on the TFI were too small for this fixation system. The back focus of the converger is inside of the converger and cannot be physically reached, requiring the reimaging lens. The reimaging lens is placed on a motorized linear stage to provide accommodation for subjects when they are asked to remove any corrective eyewear. The fixation system can accommodate up to \pm 10 diopters.

The fixation path has to pass through two tilted windows (the dichroic beamsplitters) that introduce astigmatism. Due to space constraints, one of the interferometer paths could not be folded out of plane relative to the other to compensate for the other beamsplitter. To minimize astigmatism, two additional windows of equal thickness were placed and counter-rotated out of plane relative to the dichroic beampslitters.

One of the advantages of placing the fixation assembly internal to the system is that it simplifies the test setup. The original TFI system had an external fixation assembly and required an additional step of aligning the fixation system to the subject before the interferometer system could be aligned. ${ }^{62}$ The second issue with an external fixation system was that to test both of the subject's eyes during a test, either two fixation assemblies were required, or one had to be removed and placed on the other side.

A second advantage of placing the fixation assembly in-line with the interferometer systems has to do with the placement of the aperture stop for the imaging system. The aperture stop in the fixation system is designed to be conjugate to the pupil of the human eye and is stopped down to match the nominal diameter of the human pupil in a brightly lit room. The fixation assembly is coaligned to the other two interferometer systems, such that the fixation target is only visible to the human subject when their line-of-sight is coaligned to the optical axis of the interferometer. This allows the interferometer to be repeatedly coaligned to the visual line-of-sight of the subject between measurement sessions, which was not possible with the TFI.

\subsection{Laser Safety}

A detailed laser safety analysis of the TFI has been documented by Primeau et al. ${ }^{69}$ that showed that the system was eye safe. The surface interferometer is functionally equivalent to the TFI, so the results from that study are still applicable to that subsystem. The analysis assumes a worst-case exposure: continuous laser exposure and an eye accommodation and system alignment that would produce the largest irradiance on any surface within the eye. The results were a maximum permissible exposure (MPE) of $31.4 \mathrm{~mW}$.

The cat's eye interferometer is the same configuration as the TFI that allows for the same safety analysis to be repeated, with adjustments to two key optical parameters: wavelength and $f$ /\#. Repeating the analysis of Primeau et al. for the cat's eye interferometer results in a calculated MPE of $14 \mathrm{~mW}$, a result of the slower working $f$ /\# of the cat's eye interferometer.

An additional deviation from the original analysis is the consideration of multiwavelength sources. The ANSI recommendation for multiwavelength laser emission is to derive the MPE for each wavelength and analyze the exposure limits for the cumulative values. ${ }^{70}$ A cumulative safety factor that includes both systems results in an MPE of $10 \mathrm{~mW}$ for the ICT.

Each interferometer system is designed to operate under $1 \mathrm{~mW}$ and includes a safety interlock to ensure that it does not exceed this output. The safety interlock consists of an internal power meter that monitors the laser source, as described in Secs. 3.2 and 3.3. Each power meter is calibrated to the output of the system using a photodetector with a National Institute of Standards and Technology (NIST) traceable calibration at the output of the system. The output of the internal power meter is constantly monitored to ensure that the system output does not exceed $1 \mathrm{~mW}$. In the event that the output power exceeds $1 \mathrm{~mW}$, the interlock system is tripped and the laser shutter for both systems is closed, regardless of which of the two systems tripped it. The two interferometers are set to operate at $\sim 0.5 \mathrm{~mW}$, such that the total exposed irradiance to the subject is $10 \times$ below the specified MPE. The laser safety analysis assumes that the sources are not modulated. Since both sources are modulated with duty cycles in the order of $100 \mu \mathrm{sec} / 33.3 \mathrm{~ms}=0.3 \%$ and are temporally offset (Fig. 8), actual exposure is significantly less than what the worst-case analysis shows.

\section{Data Processing}

The design of the ICT system ensures that cornea and tear film surface information can be captured. However, a number of steps are required to process the measured data into something 
that represents the absolute surface topography. A flowchart is shown in Fig. 10 that identifies key processing steps that will be discussed in more detail in this section. The first step is to phase unwrap the measured interferometric data and is discussed in Sec. 4.1. Section 4.2 describes the methods by which the absolute surface topography can be reconstructed from the measured information. Finally, Sec. 4.3 describes how the cat's eye measurement is used to support the reconstruction method.

\subsection{Phase Unwrapping}

A more detailed discussion of phase unwrapping and the methods used are discussed in the TFI reference ${ }^{62}$ but are briefly reviewed in this section. Measured interferometric data can only be recovered by a modulo of $2 \pi$. That is to say, the true phase $(\phi)$ is related to the recovered phase $(\psi)$ by a modulus or wrapping function $\mathcal{W}$

$\psi=\mathcal{W}[\phi]=\phi-2 \pi\left\lfloor\frac{\phi}{2 \pi}\right\rfloor$,

where the $\rfloor$ represents a rounding operation. Phase recovery becomes complicated when the measured phase exceeds a wavelength or $2 \pi$ in phase. When this occurs, the modulo operation "wraps" the data resulting in a discontinuity that appears in the recovered phase. There are a number of methods to remove these discontinuities, in effect recovering the true phase, which is more generally referred to as phase unwrapping..$^{71}$

The issue with phase unwrapping as it relates to the ICT is that this data is unlike most data captured in optical

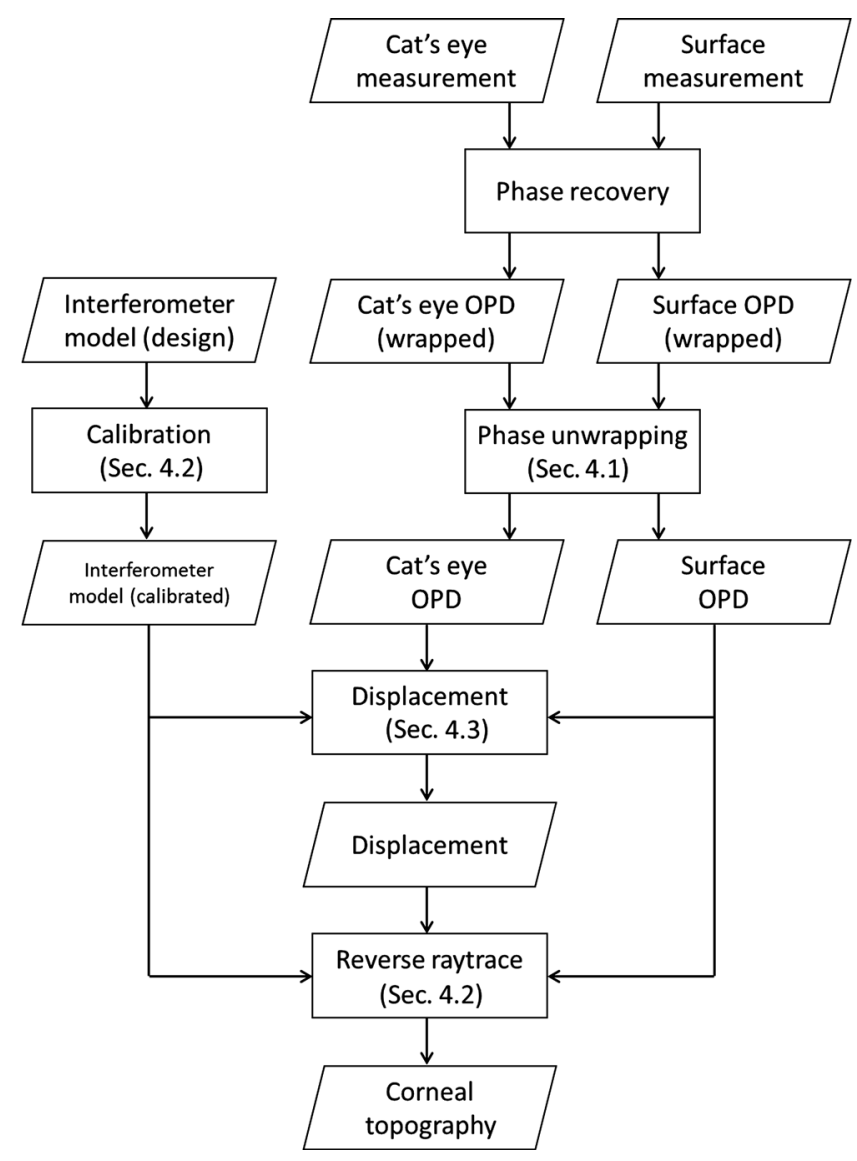

Fig. 10 Flowchart for recovering corneal topography. interferometry. Phase unwrapping routines that are used with most commercial analysis software packages that support interferometric measurements do not have to be robust (i.e., designed to handle extreme data). That is to say, most optical surfaces that are measured by commercial interferometer systems are smooth, continuous, and low-noise measurements that present little challenge to most phase unwrapping routines. Measurements with the ICT system are a challenge.

First, the converger optical assembly is designed to null against the shape of the average human cornea. The cornea varies across the population, including variations in base curvature, asphericity, axial symmetry, and higher-order surface features (refer to Sec. 2.1). Furthermore, the tear film surface is a dynamic and turbulent surface. Every blink forms a tear film surface that rapidly evolves. Surface tension will pull some of the structure upward against gravity and exposure to air will begin drying out the tear film. Breakup areas where the tear film no longer binds to the cornea will result in surface height changes that are in the order of the tear film thickness. In addition to changing the overall shape of the tear film, the contact lens material will interact with the tear film and alter the dynamic behavior of the tear film.

The combination of all these factors results in complex and dense fringe patterns, which are extremely difficult to process. Two measurements that are representative of most of the measurements made with the ICT are shown in Fig. 11. These measurements are typical for bare eye measurements [Fig. 11(a)] and with contact lenses [Fig. 11(b)]. The bare eye measurement is dominated by eye motion (i.e., a misalignment of the eye with respect to the interferometer), which results in mostly power and tilt. The horizontal ridge that appears in the tear film is a common artifact that is the result of a partial blink. ${ }^{72}$ The circular drop-out zones that are approximately a few fringes in diameter are mucin globules that are suspended in the tear film. The contact lens measurement contains a lot of tilt from eye motion and contact lens motion as it settles after a blink, but the interaction of the tear film with the contact lens material results in significant complex high spatial-frequency structure. Bare eye measurements are typically processed with a quality-guided phase unwrapping method and contact lens measurements are processed with a preconditioned conjugate gradient method as described in Refs. 62 and 73.

\subsection{Surface Reconstruction}

Once the unwrapped phase is recovered from the surface and cat's eye measurements, the absolute surface topography can

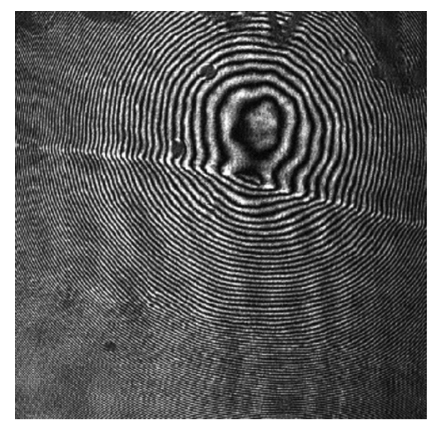

(a)

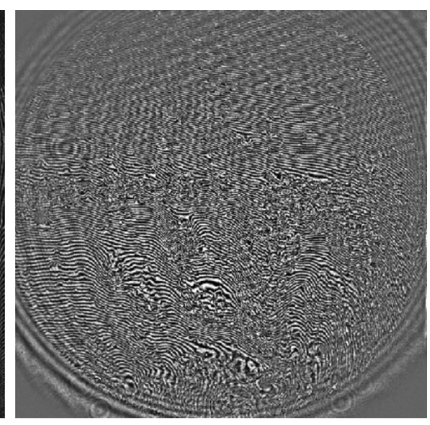

(b)
Fig. 11 Interferograms for two surface measurements of a human eye. (a) Bare eye. (b) Subject wearing a contact lens. 
be reconstructed. The reconstruction process is nontrivial; a consequence of the nonnull configuration of these measurements. Nonnull interferometry introduces additional interferometer errors, commonly known as retrace errors. ${ }^{74}$ In other words, the recovered phase from a nonnull measurement is not a direct representation of the surface deviations from an ideal surface. Examples in literature have shown that retrace errors can exceed $10 \%$ of the total surface departure from a reference shape or wavefront. $^{75,76}$ The combination of eye motion, anatomical variation, and tear film dynamics results in an average of $100 \mu \mathrm{m}$ of measured surface error and can exceed the dynamic range of the surface interferometer which is $265 \mu \mathrm{m} .{ }^{62}$ If the absolute surface topography is to be recovered with any accuracy, retrace error has to be corrected. The process for retrace error correction that is used with this system is documented in a separate publication, ${ }^{77}$ but is briefly summarized in this section.

Retrace error is introduced as a result of the perturbed test wavefront propagating through different portions of the interferometer optics than what was intended (i.e., the null path). The name originates from the geometric perspective that nonnull rays no longer trace the same path as null rays through the interferometer optics. ${ }^{74}$ An interferometer could be designed to accommodate nonnull rays and to minimize retrace errors system. ${ }^{78}$ However, nonnull interferometry on most systems, including the ICT, is overdetermined, and a straightforward solution does not exist that could compensate for all retrace errors. The result is that retrace errors have to be backed out of the measurement. The method by which this is accomplished is by simulating the propagation of the wavefront in reverse from the observation plane (e.g., detector) to the test surface. Ray tracing software is typically used to simulate the propagation and is therefore referred to as reverse ray tracing. Retrace errors did not need to be treated in the TFI because only deviations of the tear film surface from a best-fit surface were reported.

Reverse ray tracing requires two pieces of information: the measured test wavefront and an accurate model of the interferometer. The test wavefront can be determined directly from the unwrapped phase (Sec. 4.1). An accurate model of the interferometer has to be determined through a calibration process. In a null configuration, most misalignments and uncertainties in the system can be accounted for by compensating alignments or by subtracting reference measurements. When the model is used for reverse ray tracing a nonnull measurement, small differences between the model and real system can quickly accumulate errors in the reverse ray tracing process and invalidate the whole process as means to correct for retrace errors.

The process by which the interferometer model is calibrated is known as reverse optimization. ${ }^{79-81}$ Reverse optimization starts with a model of an optical system that compares measured data to data simulated in the model. An optimization routine minimizes the differences between the measured and modeled data by adjusting variables within the optical model. These variables typically represent misalignments within the system, although they can be extended to represent manufacturing residuals or environmental factors. The solution that minimizes the differences represents the state of the system. Typically, the results of a reverse optimization step are used as feedback to adjust or align components within the optical system to meet the performance requirements of the system. When applied to nonnull interferometry using reverse ray tracing, it is only necessary to have knowledge of the state of the interferometer optics.
An example of retrace error and the results of reverse ray tracing are found in the publication, ${ }^{77}$ but is briefly presented here. A test surface was diamond-turned out of brass and is in the form of a generalized biconic surface that simulates the surface of a human cornea. The sag $(Z)$ of a generalized biconic surface in cylindrical coordinates $(r, \theta)$ is

$$
Z(r, \theta)=\frac{\frac{r^{2} \cos ^{2}\left(\theta-\theta_{z}\right)}{R_{x}}+\frac{r^{2} \sin ^{2}\left(\theta-\theta_{z}\right)}{R_{y}}}{1+\sqrt{1-\frac{\left(1+K_{x}\right) r^{2} \cos ^{2}\left(\theta-\theta_{z}\right)}{R_{x}^{2}}-\frac{\left(1+K_{y}\right) r^{2} \sin ^{2}\left(\theta-\theta_{z}\right)}{R_{y}^{2}}}},
$$

where the radius of curvature $R_{x}$ and conic constant $K_{x}$ are along the $\theta_{z}$ meridian, and the radius $R_{y}$ and conic constant $K_{y}$ are along the orthogonal meridian. The parameters for the test surface used for this demonstration are $R_{x}=-8.00 \mathrm{~mm}$, $R_{y}=-8.10 \mathrm{~mm}, K_{x}=-0.25$, and $K_{y}=+0.50$. The test configuration resulted in the surface being rotated by $\theta_{z}=8.1 \mathrm{deg}$. The surface was nominally aligned to the interferometer then laterally translated by $75 \mu \mathrm{m}$ and defocused by $150 \mu \mathrm{m}$ to simulate the average range of eye motion that occurs during testing with the ICT. The measured OPD is $95 \mu \mathrm{m} \mathrm{PV}$, which consists mostly of tilt and defocus resulting from the misalignment (Fig. 12).

The amount of retrace error that is introduced by the test surface in this configuration is $12.7 \mu \mathrm{m}$ PV and $2.7 \mu \mathrm{m}$ root-meansquare (RMS) [Fig. 13(a)]. The retrace error is determined by adding the measured OPD (double-pass and parity corrected) to a model of the nominal test surface that has been updated with the measured radius of curvature from the cat's eye measurement (discussed in more detail in Sec. 4.3). The difference between the reconstructed test surface and known biconic shape is the amount of retrace error that is shown in Fig. 13(a).

The residual error when using reverse ray tracing results in $67 \mathrm{~nm}$ PV and $14 \mathrm{~nm}$ RMS and is shown on the right in Fig. 13. The residual error is a combination of interferometer errors and test surface errors. The residual interferometer errors are a result of an incomplete interferometer calibration (i.e., uncharacterized surfaces and component alignments), stray light (spurious reflections), and fringe print-through (phase unwrapping errors).

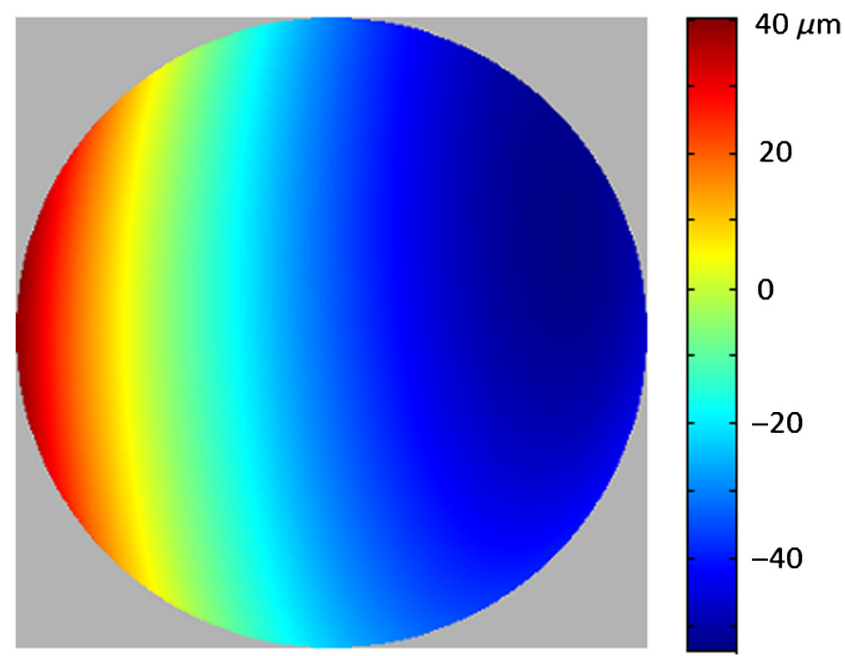

Fig. 12 Measured OPD of the brass biconic surface. Surface has been laterally translated by $75 \mu \mathrm{m}$ and defocused by $150 \mu \mathrm{m} ; \mathrm{PV}$ $95 \mu \mathrm{m}$. 


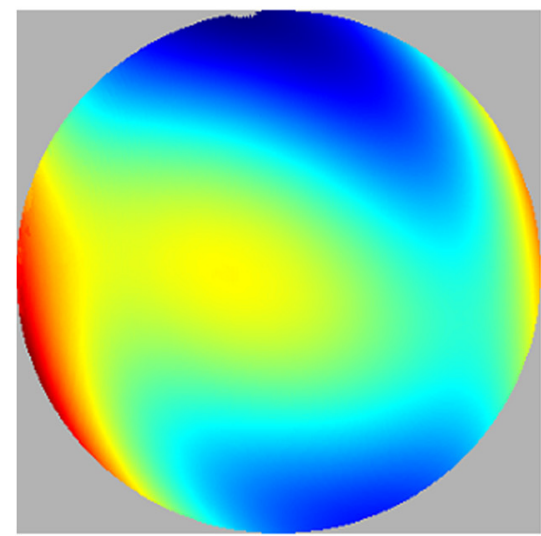

(a)

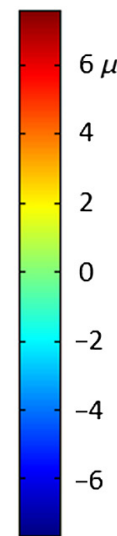

$6 \mu \mathrm{m}$
4
2
0
-2
-4
-6

\begin{abstract}
.
\end{abstract}
Fig. 13 (a) Retrace errors (PV $12.6 \mu \mathrm{m}$, RMS 2.7 $67 \mathrm{~nm}$, RMS $14 \mathrm{~nm}$ ). Display area is $6 \times 6 \mathrm{~mm}$.

If additional reconstruction accuracy is required, the surfaces within the partial null lens will need to be characterized in addition to minimizing error from some of the other previously mentioned effects. However, the residual errors are within the specifications of the ICT, so further corrections were not pursued.

\subsection{Displacement Calculation}

A detail that was briefly discussed in Sec. 4.3 was the cat's eye measurement. The cat's eye interferometer provides a secondary piece of information that is necessary to reconstruct the absolute topography of the surface being tested. In an ideal null reflection test of a spherical surface, the radius of curvature could be measured by translating the surface from a cat's eye position to a confocal position and recording the distance. This distance would directly correspond to the radius of curvature. In a nonnull measurement, the distance may no longer directly relate to a radius of curvature or may not be accessible. Instead, the surface reconstruction method describes this as a displacement measurement that supports the reverse ray trace operation. ${ }^{77}$ It provides an absolute position of the test surface relative to the interferometer or to a known point in space (e.g., the focus of the transmission sphere). In effect, the displacement measurement determines how far the test wavefront needs to propagate in the reverse ray trace operation to recover the surface topography.

The simultaneous measurement with the cat's eye interferometer is important for this instrument because of the dynamic environment. Eye motion and the evolution of the tear film structure following a blink does not allow for a traditional cat's eye to confocal measurement. Another complication of the cat's eye measurement is that it also contains retrace errors, which would require a calibration of the cat's eye interferometer. However, the output of the cat's eye measurement is much less complicated when compared to the surface interferometer output. A cat's eye measurement provides a single value-a displacement. This is unlike the surface interferometer, which has to resolve surface topography that is one-to-one with the resolution of the phase measurement (i.e., each pixel represents a different measurement). A first-order approximation for displacement could be derived from the amount of defocus measured in the cat's eye measurement $\delta z \approx 4(f / \#)^{2} \mathrm{Z}_{3}$,

where $\delta z$ is the amount of translation along the optical axis, $Z_{3}=2 \rho^{2}-1$ is the Zernike polynomial term for defocus, and $f / \#$ is the working $f / \#$ of the cat's eye interferometer. In practice, determination of the displacement of the test surface will not be as straightforward. Ocular variation and eye motion will result in large displacements of the corneal surface from the cat's eye focus. The convex and irregular shape of the eye, coupled with these large motions, will result in a distortion of the cat's eye wavefront that will skew the displacement calculation. The result is that the calculated displacement will have to be a function of measurements from both interferometer subsystems:

$\delta z=f\left(\phi_{\text {surf }}, \phi_{\text {cat }}\right)$

where $\phi_{\text {surf }}$ and $\phi_{\text {cat }}$ are the measured optical path differences from the surface and cat's eye interferometers, respectively. The measured optical path differences can be described by the coefficients of a Zernike polynomial fit, which can in turn be used to describe the displacement through a polynomial fit. The relationship that was empirically derived for the ICT system is

$$
\begin{aligned}
\delta z= & a_{1}+a_{2} C_{3}+a_{3} C_{3}^{2}+a_{4} S_{3}+a_{5} S_{3}^{3}+a_{6} S_{3}^{4}+a_{7} S_{1} \\
& +a_{8} S_{2}+a_{9} S_{6}+a_{10} S_{7}
\end{aligned}
$$

where $a_{i}$ are the polynomial coefficients for the displacement function and $C_{j}$ and $S_{j}$ represent the $j$-th Zernike polynomial coefficients for the cat's eye and surface measurements, respectively.

Calibration of the cat's eye interferometer is accomplished by fitting the polynomial against a set of measurements of known surfaces that vary in shape and alignment. The process of recovering the displacement measurement requires fitting Zernike polynomials to the surface and cat's eye measurements; the coefficients are then passed as inputs into the displacement equation [Eq. (5)]. The displacement measurement is a required input to the surface reconstruction methods described in Sec. 4.2, which can recover the absolute surface topography of any shape that is resolvable by the surface interferometer. 


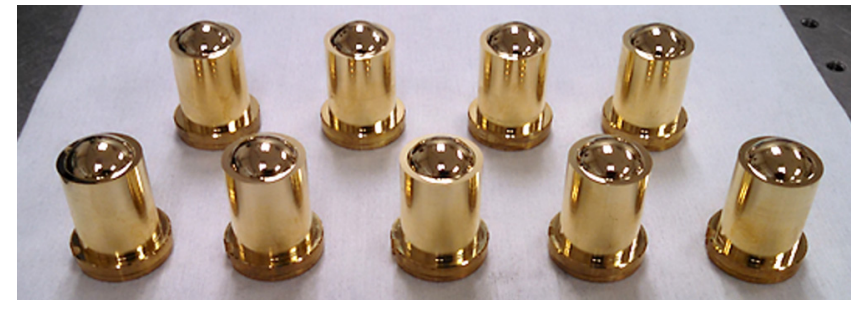

Fig. 14 Image of the cat's eye calibration test surfaces.

\section{Results}

A reference corneal model or a "golden surface," was manufactured to simulate the nominal shape of the cornea. The surface has a 7.8 -mm radius of curvature and a -0.25 conic constant. The surface was manufactured by QED (Rochester, New York) and has a figure error of $\lambda / 20 \mathrm{PV}$ and $\lambda / 100 \mathrm{RMS}$ $(\lambda=632.8 \mathrm{~nm})$ over a $7-\mathrm{mm}$ clear aperture. The golden surface was used to separately align the partial null optics and to align and verify both interferometer systems in the ICT. Once the system was aligned, the golden surface was used to calibrate the surface interferometer model by the methods described in Sec. 4.2.

Diamond-turned brass surfaces were manufactured for calibration of the cat's eye interferometer and verification of the ICT system performance. The brass surfaces are generalized biconic surfaces described by Eq. (2). The set of brass surfaces used for the cat's eye calibration are shown in Fig. 14 and the surface parameters are given in Table 1. The other half of the brass surfaces listed in Table 1 are designed to independently verify the ICT system. The distribution of surface parameters was specified to match $99 \%( \pm 3 \sigma)$ of the population (Sec. 2.1). Note that the parameters of surface 010 are identical to surface 001 . This was a manufacturing error and discovered during the testing described in this section-the surface was supposed to have a radius of $-8.00 \mathrm{~mm}$ and a -1.00 conic constant. Measurements for surface 010 are still included in the results.

\subsection{Performance}

Performance of the ICT is primarily verified with the last 10 surfaces listed in Table 1. The cat's eye calibration surfaces are used to generate the coefficients for displacement [Eq. (5)] but are also included as part of this section because they provide additional data points of comparison between the ICT and other systems. A commercially available corneal topographer was included in the testing. The device is a Keratron Picollo (Optikon. Roma, Italy), which is a computerized Placido disc topographer (refer to Sec. 1). The system uses an optical trip line to determine the distance from the corneal vertex to the device, which is referred to by the manufacturer as an eye positioning control system (EPCS). The manufacturer also claims that the EPCS has the ability to correct for decentration of the cornea with respect to the optical axis of the imaging system.

A total of 35 measurements per brass surface were made with the ICT. First, each brass surface was nulled or optimally aligned to the surface interferometer. Next, the brass surface was translated $\pm 75 \mu \mathrm{m}$ along the $x$ - and $y$-axis (lateral translations) for an additional four measurements. The value of $75 \mu \mathrm{m}$ was chosen based on previous measurements made with the TFI system; it is the observed average displacement of an eye during measurements. The surface was then translated by $+75 \mu \mathrm{m}$ along
Table 1 Parameters for brass test surfaces.

\begin{tabular}{|c|c|c|c|c|c|}
\hline Part ID & $R x$ & Ry & $K x$ & $K y$ & Usage \\
\hline 001 & -7.00 & -7.00 & -0.25 & -0.25 & $\begin{array}{l}\text { Cat's eye } \\
\text { calibration }\end{array}$ \\
\hline 002 & -7.25 & -7.25 & -0.25 & -0.25 & \\
\hline 003 & -7.50 & -7.50 & -0.25 & -0.25 & \\
\hline 004 & -7.75 & -7.75 & -0.25 & -0.25 & \\
\hline 005 & -8.00 & -8.00 & -0.25 & -0.25 & \\
\hline 006 & -8.25 & -8.25 & -0.25 & -0.25 & \\
\hline 007 & -8.50 & -8.50 & -0.25 & -0.25 & \\
\hline 008 & -8.75 & -8.75 & -0.25 & -0.25 & \\
\hline 009 & -9.00 & -9.00 & -0.25 & -0.25 & \\
\hline 010 & -7.00 & -7.00 & -0.25 & -0.25 & $\begin{array}{c}I C T \\
\text { verification }\end{array}$ \\
\hline 011 & -8.00 & -8.00 & -0.50 & -0.50 & \\
\hline 012 & -8.00 & -8.00 & 0.00 & 0.00 & \\
\hline 013 & -8.00 & -8.00 & 0.50 & 0.50 & \\
\hline 014 & -8.00 & -8.00 & 1.00 & 1.00 & \\
\hline 101 & -7.00 & -7.15 & -0.25 & -0.25 & \\
\hline 102 & -8.00 & -8.20 & -0.25 & -0.25 & \\
\hline 103 & -8.80 & -9.00 & -0.25 & -0.25 & \\
\hline 104 & -8.00 & -8.10 & -0.25 & 0.25 & \\
\hline 105 & -8.00 & -8.10 & -0.25 & 0.50 & \\
\hline
\end{tabular}

the $z$-axis and the same five measurements were repeated. The process was repeated for three steps along the $+z$-direction, then three steps along the $-z$-direction (i.e., relative to the null position) for a total of 35 measurements. The same process was used for the Keratron Picollo system, with exception of the $z$-axis translation. The Keratron Picollo relies on the optical trip line to detect the position of the eye at fixed axial location. Therefore, in order to test a comparable number of data points, the surface was translated into multiple locations within a $150-\mu \mathrm{m} \times 150-\mu \mathrm{m}$ area centered on the optical axis of the device. The results of these measurements are summarized in Table 2, presented as a bias $\pm 1 \sigma$. In general, it can be seen that the ICT system outperforms the Keratron Picollo by at least an order of magnitude.

For each measurement, a generalized biconic surface was fit to the data to estimate the radius and conic constant of the surface. The fitting routine allowed the surface to tip and tilt to accommodate small alignment errors of the surface relative to the device. Corneal power was calculated from the recovered radius as

$\Phi=\frac{n_{k}-1}{R}$ 
Table 2 Performance comparison of the ICT to the Keratron Piccolo.

\begin{tabular}{|c|c|c|}
\hline Accuracy & ICT & Keratron Piccolo \\
\hline Radius $(\mu \mathrm{m})$ & $3.1 \pm 4.2$ & $-25.1 \pm 50.8$ \\
\hline Conic & $0.0001 \pm 0.0091$ & $0.0413 \pm 0.0653$ \\
\hline Power (D) & $-0.0166 \pm 0.0224$ & $0.1344 \pm 0.2673$ \\
\hline Astigmatism (D) & $0.0010 \pm 0.0086$ & $-0.0732 \pm 0.4597$ \\
\hline Residual fit RMS (nm) & $25.2(<112.3)$ & $305.5(<886.8)$ \\
\hline Residual fit $\mathrm{PV}_{98}(\mathrm{~nm})$ & $129.1(<607.9)$ & $1503.7(<4339.1)$ \\
\hline Spatial resolution $(\mu \mathrm{m})$ & 6 & $>100$ \\
\hline
\end{tabular}

where $n_{k}=1.3375$ is the keratometric index of refraction; an effective index used when describing the total power of the cornea resulting from an anterior surface measurement and accounting for the negative power introduced by the posterior surface. Astigmatism is defined as the difference in corneal power along the two meridians of the biconic surface. Spatial resolution is determined by the imaging system parameters for each system, but was independently verified by measuring known targets with calibrated feature sizes.

Measurement results are presented graphically in Figs. 15-17 using box and whisker plots. The $y$-axis is the error in the estimation of the parameter. The box height represents the RMS error and is centered about the mean. The whiskers represent the maximum and minimum estimation errors for that parameter. In addition to the ICT and Keratron Piccolo systems, another set of measurements are shown where the surface interferometer data and the cat's eye data are used independently. These results

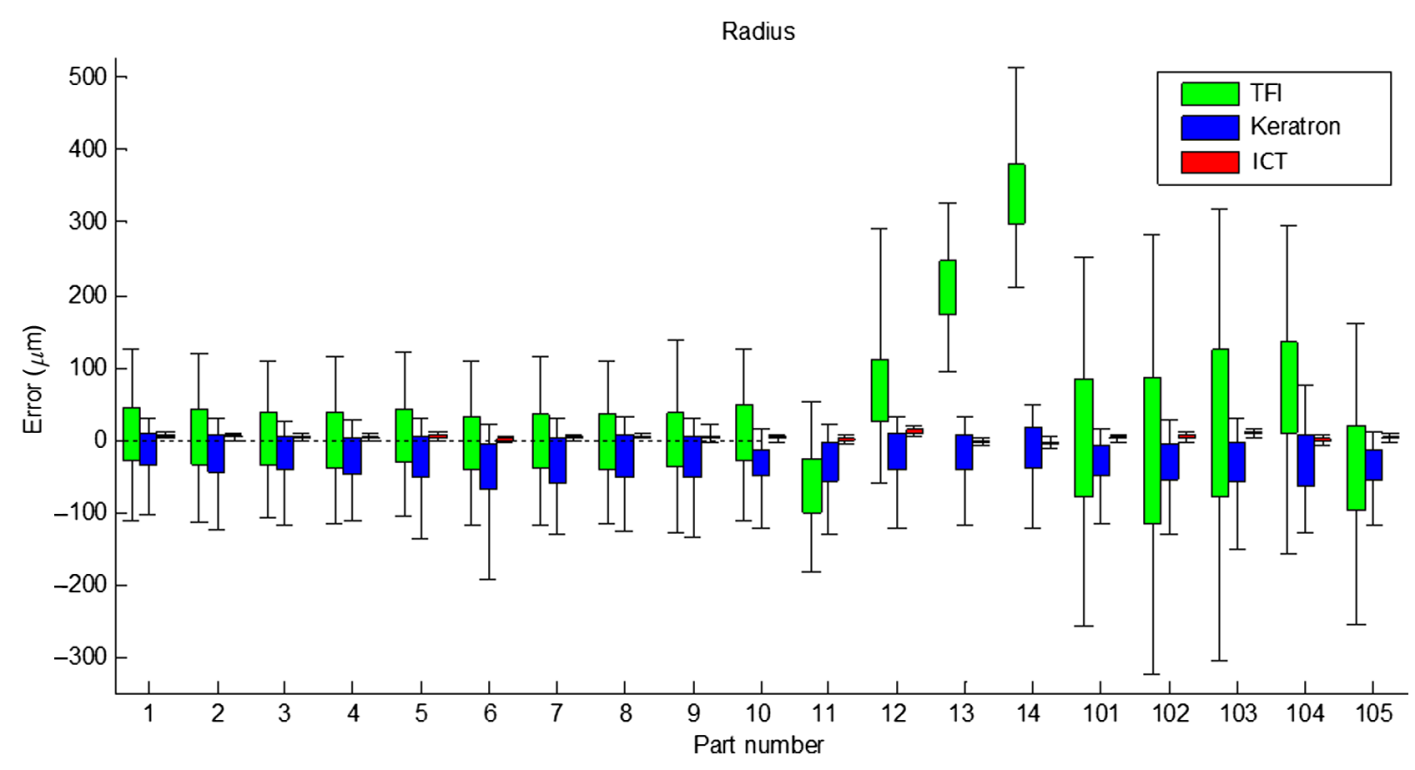

Fig. 15 Box and whisker plot comparison of the TFI, ICT, and Keratron Piccolo for estimating radius of curvature.

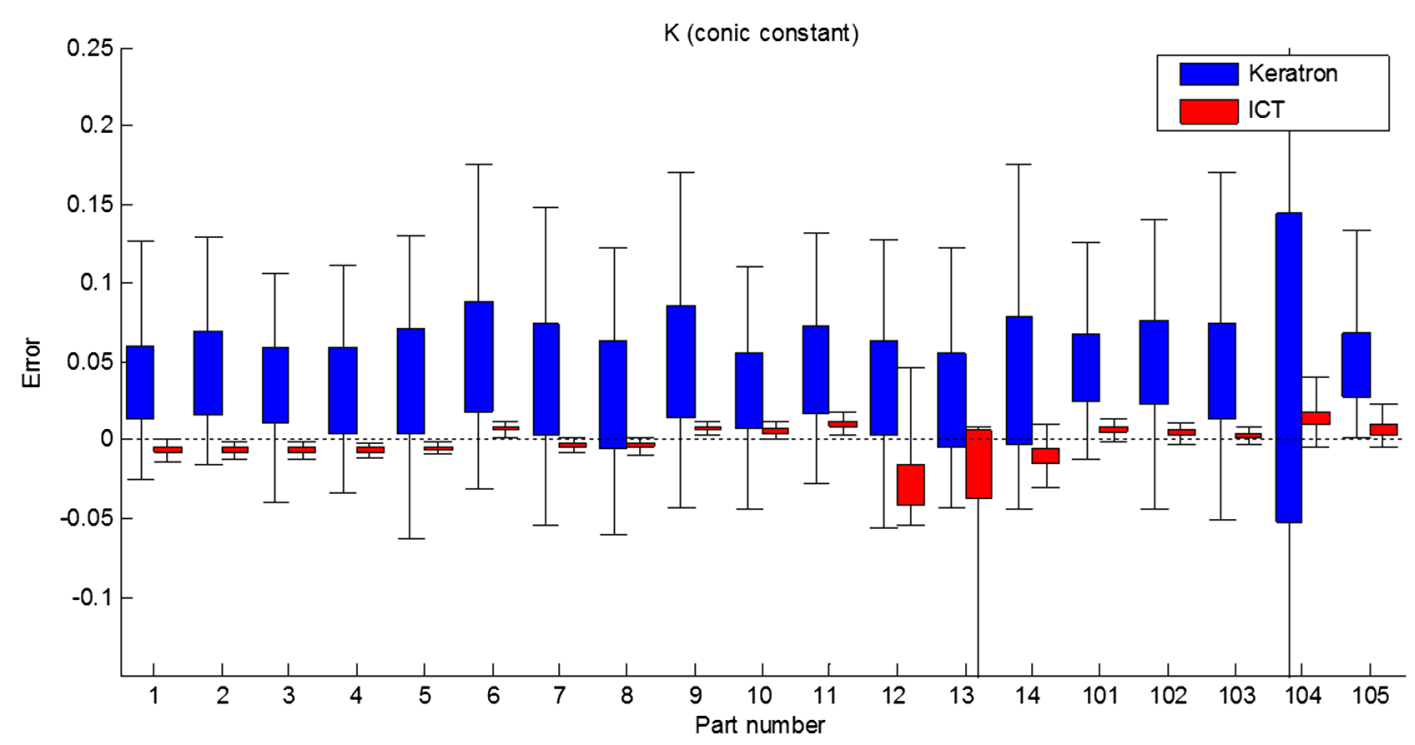

Fig. 16 Box and whisker plot comparison of the ICT and Keratron Piccolo for estimating the conic constant. 
(labeled TFI) demonstrate what would happen if the ICT measurements were not corrected for retrace error. The cat's eye measurement can be converted directly into a radius of curvature by repurposing Eq. (5) and this radius measurement is used to update the nominal corneal model. The surface measurements are mapped onto this corneal model.

Figure 15 shows the error in estimation of the radius of curvature for the three systems (TFI, Keratron, and ICT). In general, the ICT system outperforms either system by at least an order of magnitude. The TFI results behave similarly to the Keratron Picollo for surfaces 001 through 010, because the surface shapes are relatively simple. The first 10 surfaces were designed with the same nominal conic constant $(-0.25)$ and only the radius of curvature is changed. The reason that the TFI performs poorly for these surfaces, when compared to the ICT, is due to the retrace errors that occur during motion of the surfaces. However, the remaining surfaces have varying amounts of conic constant or nonrotationally symmetric features, at which point the TFI performance becomes worse than the Keratron Piccolo. Despite the fact that the Keratron Piccolo is calibrated, it underestimates the radius of curvature for all of the measurements. This is because most of the measurements were made with some decentration relative to the optical axis that introduced errors in the recovered topography, despite the manufacturer's claim that this would be corrected.

The plots shown for the estimation errors of the conic constant (Fig. 16) are similar to the radius plots. TFI errors are not shown because a number of results exceed the plot scale and the previous example is sufficient to demonstrate that the TFI system is incapable of measuring absolute surface shape parameters. A final comparison plot is the biconic fit residuals (Fig. 17), which shows that the Keratron Picollo produces large residual errors. As will be shown in Sec. 5.2, residual errors will limit the Keratron Piccolo from resolving higher spatial-frequency topography and most of the tear film structure.

An additional improvement of the ICT system over the prior TFI is that the reconstructed absolute surface topography results in a corrected plate scale (i.e., magnification). The absence of this information from TFI measurements resulted in an uncertainty of feature dimensions by as much as $40 \%$.

\subsection{Human Subjects Testing}

The ICT was approved for human subjects testing by the University of Arizona Institutional Review Board and adheres to the tenants of the Declaration of Helsinki. Informed consent is obtained from a subject prior to testing. Testing was performed on healthy adults, aged 18 to 60 years. Subjects were not allowed to participate if they had any known eye disease or have undergone refractive surgery. Subjects outside of this age range or with an eye disease may introduce complications that inhibit the ICT system's ability to collect data. Nine human subjects were examined using the ICT. A trained operator can align the system to a subject in under a minute. Acquisition times are limited only by the subjects' ability to comfortably maintain fixation. Collection times are generally limited to $5 \mathrm{~min}$ before a break is given to the subject to minimize fatigue.

The results from human subjects testing are presented in this section. Temporal surface measurements (e.g., topographic movies) are possible with this system and has been previously demonstrated with tear film measurements. ${ }^{62}$ Static data (i.e., single-frame snapshots) are presented here to focus on the absolute surface reconstruction capabilities of the system. Comparisons to the Keratron Piccolo are also presented.

\subsubsection{Data presentation}

This section discusses the different data presentation formats that will be used in the following sections. Raw topographic data are not displayed as it is dominated by the overall sag of the corneal surface, which is in the order of $0.5 \mathrm{~mm}$ and provides little feedback. The first display format that is used is the conic removed surface. This is the residual from the best-fit conic to the topographic data, where the conic surface is defined as

$Z_{\text {conic }}(r)=\frac{r^{2} / R}{1+\sqrt{1-(K+1) r^{2} / R^{2}}}$,

where $R$ is the radius of curvature, $K$ is the conic constant, and $r$ is the radial coordinate. The conic removed surface is useful for

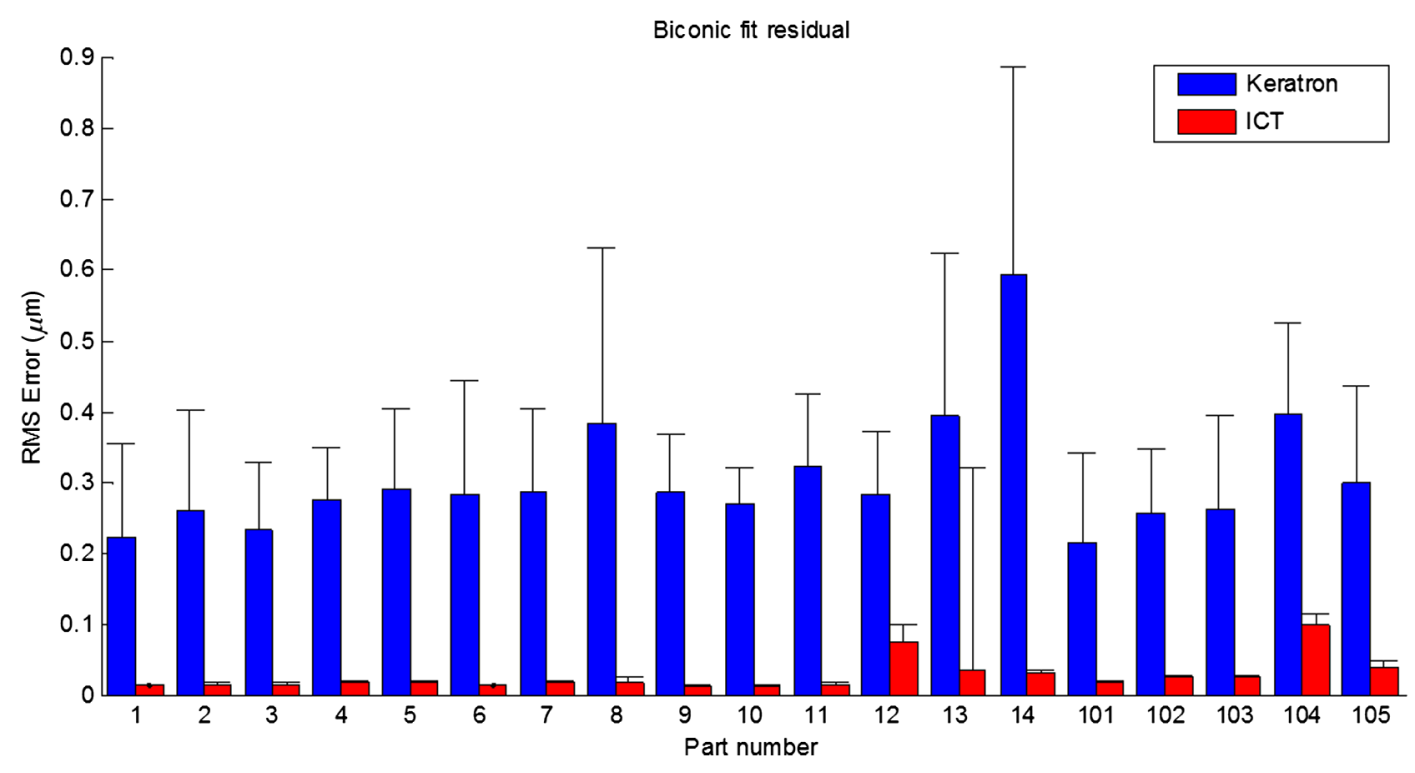

Fig. 17 Box and whisker plot comparison of the ICT and Keratron Piccolo for biconic fit residuals. 
displaying nonrotationally symmetric features of the corneal topography, typically astigmatism.

The next surface is the biconic removed surface. This is the residual from the best-fit generalized biconic that is described by Eq. (2). The generalized biconic fit parameters are the most useful metric for describing the overall topography of the cornea. The residual surface is a combination of the remaining topography and tear film structure. Half of the subjects measured contained residual topographic structure that was not adequately described by the biconic; generally, these structures showed up as trefoil, quadrafoil, or more complex surface shapes. In these instances, it is useful to look at the residual surface created by subtracting a 15-term Zernike polynomial. This final surface, the Zernike removed surface, is predominantly tear film structure, which contains mid to high spatial-frequency structure. It should be noted that none of these residual surfaces completely isolates the tear film structure from the cornea structure. This would require either a simultaneous measurement of the tear film thickness or analyzing the dynamics of the tear film and isolating the constant structure that would represent the corneal topography.

\subsubsection{Subject $A$}

The first subject that is presented was chosen because their cornea contained enough unique topography that spans low to mid spatial-frequencies that would provide a good comparison between the ICT and the Keratron Piccolo. Generalized biconic fit results from a set of measurements from both systems shows good agreement (Table 3). The surface interferograms from the ICT for this example set are shown in Fig. 18; they are dominated by tilt as a result of eye motion. The conic removed surface from both systems shows comparable amounts of corneal astigmatism (Fig. 19). The biconic removed surface also shows good agreement (Fig. 20), but the mid to high spatial-frequency structure is only resolvable in the ICT measurement. It should be further noted that the biconic removed surface for the Keratron Piccolo is close to the limit of the resolution of the system, as demonstrated by previous results (Sec. 5.1). In other words, if the residual surface had been any flatter (i.e., $<1.5 \mu \mathrm{m}$ PV), the results from the Keratron Piccolo would be mostly noise as seen in the Zernike removed surfaces (Fig. 21). The ICT system has no issue resolving the mid to high spatial-frequency structure, which is primarily tear film structure.

\subsubsection{Subject $B$}

This subject exhibited relatively high amounts of corneal astigmatism and also wore toric contact lenses to correct for visual

Table 3 Subject A, biconic fit results.

\begin{tabular}{lccccc}
\hline & \multicolumn{2}{c}{ Left } & & \multicolumn{2}{c}{ Right } \\
\cline { 2 - 3 } \cline { 5 - 6 } Parameter & ICT & Keratron & & ICT & Keratron \\
\hline$R x(\mathrm{~mm})$ & -7.708 & -7.594 & & -7.597 & -7.577 \\
$R y(\mathrm{~mm})$ & -7.441 & -7.339 & & -7.369 & -7.339 \\
$K x$ & -0.334 & -0.248 & & -0.320 & -0.174 \\
$K y$ & -0.318 & -0.285 & & -0.388 & -0.237 \\
$\theta(\mathrm{deg})$ & -8.08 & -11.56 & & 2.89 & 5.77 \\
\hline
\end{tabular}

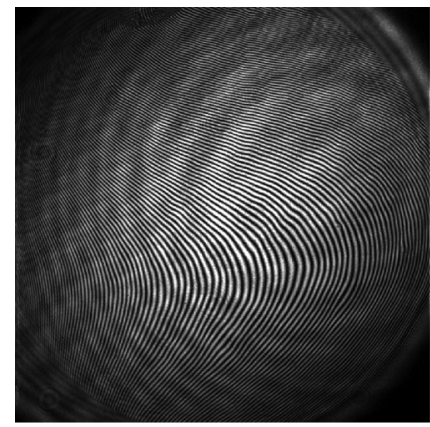

(a)

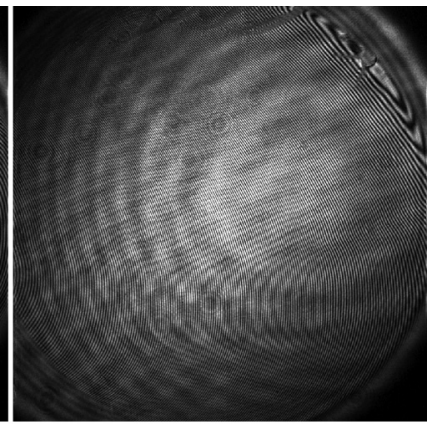

(b)
Fig. 18 Subject A, surface interferograms. (a) Left eye. (b) Right eye.

astigmatism. The contact lenses were Biofinity toric by CooperVision. The left eye prescription was $+0.5 \mathrm{D} /-2.25 \mathrm{D} \times$ $10 \mathrm{deg}$ and the right eye prescription was $+0.25 \mathrm{D} /-1.75 \times$ $170 \mathrm{deg}$.

Bare eye. Measurements were first made on the subject's bare eye. Biconic fit results for the bare eye is shown in Table 4. Surface interferograms from the ICT are shown in Fig. 22. The conic removed surfaces (Fig. 23) shows the large amount of corneal astigmatism present with this subject. The general astigmatic shape appears slightly different between the ICT and the Keratron Piccolo. However, it is known that the reconstruction method used by the Keratron Piccolo is susceptible to errors for nonrotationally symmetric surfaces. ${ }^{82}$ Therefore, it is more likely that the ICT surfaces provide a more correct representation of the surface, which is also supported by the performance results presented in Sec. 5.1.

The biconic removed surfaces (Fig. 24) show better correlation between the two topographer systems, which is dominated by low to mid spatial-frequency structure. The Zernike removed

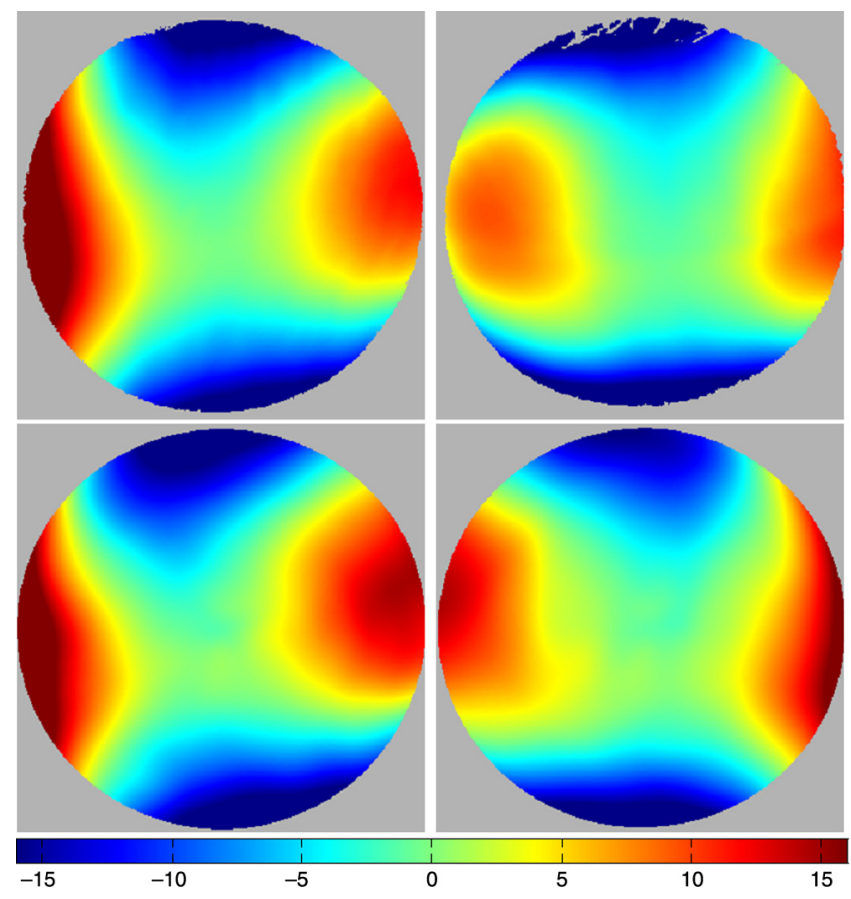

Fig. 19 Subject A, conic removed corneal surface height. Left eye left column, right eye right column, ICT top row, Keratron Piccolo bottom row. Display area is $6 \times 6 \mathrm{~mm} ; \pm 16.0-\mu \mathrm{m}$ color height scale; red is away from eye. 

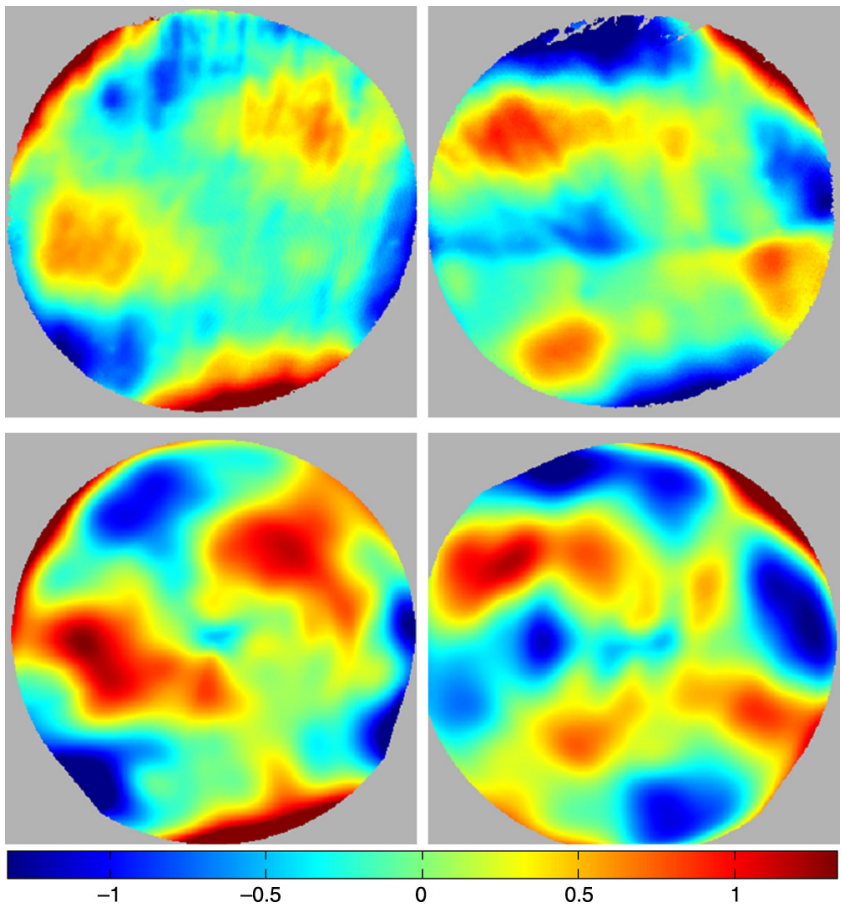

Fig. 20 Subject $A$, biconic removed corneal surface height. Left eye left column, right eye right column, ICT top row, Keratron Piccolo bottom row. Display area is $6 \times 6 \mathrm{~mm} ; \pm 1.25-\mu \mathrm{m}$ color height scale; red is away from eye.

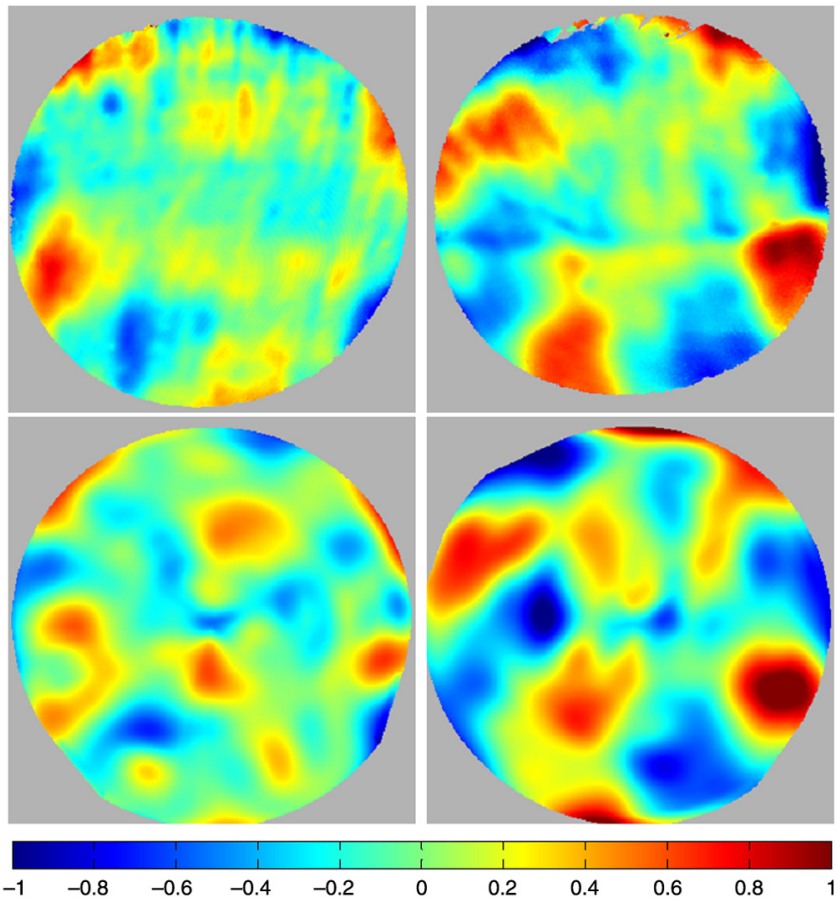

Fig. 21 Subject A, Zernike removed corneal surface height. Left eye left column, right eye right column, ICT top row, Keratron Piccolo bottom row. Display area is $6 \times 6 \mathrm{~mm} ; \pm 1.0-\mu \mathrm{m}$ color height scale; red is away from eye. About 15 Zernike terms removed from surface.

surfaces (Fig. 25) display what appears to be mostly tear film structure, but the residual pentafoil that appears in the ICT measurements is residual topography. The scale of the Zernike removed surface for the Keratron Piccolo is less than the
Table 4 Subject B, biconic fit results.

\begin{tabular}{lccccc}
\hline \multirow{2}{*}{ Parameters } & \multicolumn{2}{c}{ Left } & & \multicolumn{2}{c}{ Right } \\
\cline { 2 - 3 } \cline { 5 - 6 } \cline { 5 - 6 } R & ICT & Keratron & & ICT & Keratron \\
$R y(\mathrm{~mm})$ & -8.011 & -7.992 & & -8.078 & -8.104 \\
$K x$ & -7.471 & -7.604 & -7.622 & -7.669 \\
$K y$ & -0.143 & -0.242 & & -0.329 & -0.201 \\
$\theta(\mathrm{deg})$ & -0.118 & 0.146 & & 0.102 & 0.042 \\
\hline & -4.79 & -8.22 & & 2.38 & 5.50 \\
\hline
\end{tabular}

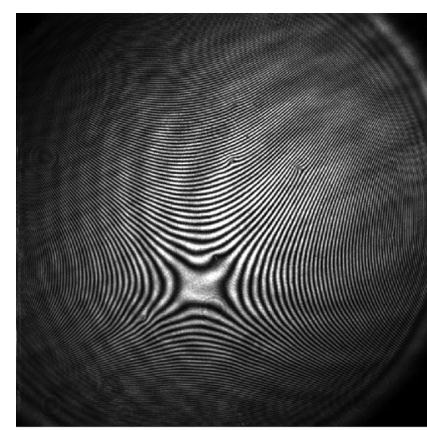

(a)

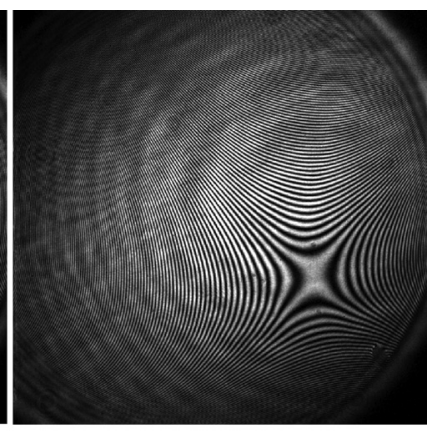

(b)
Fig. 22 Subject B, surface interferograms on bare eye. (a) Left eye. (b) Right eye.

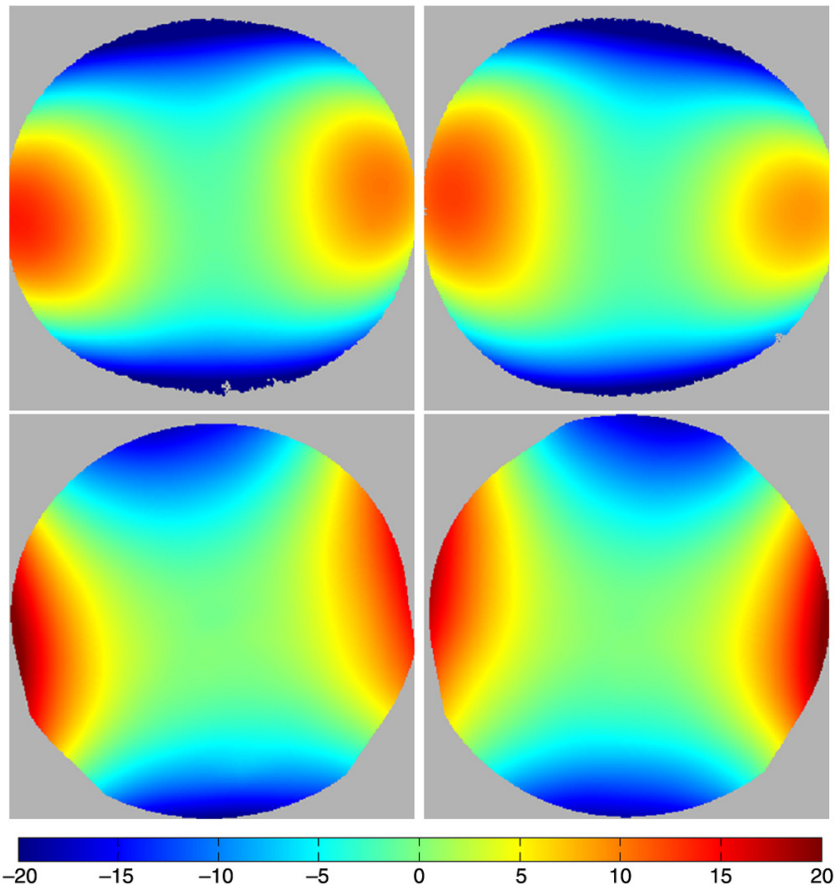

Fig. 23 Subject $B$, conic removed corneal surface height (bare eye). Left eye left column, right eye right column, ICT top row, Keratron Piccolo bottom row. Display area is $6 \times 6 \mathrm{~mm} ; \pm 20.0-\mu \mathrm{m}$ color height scale; red is away from eye. 


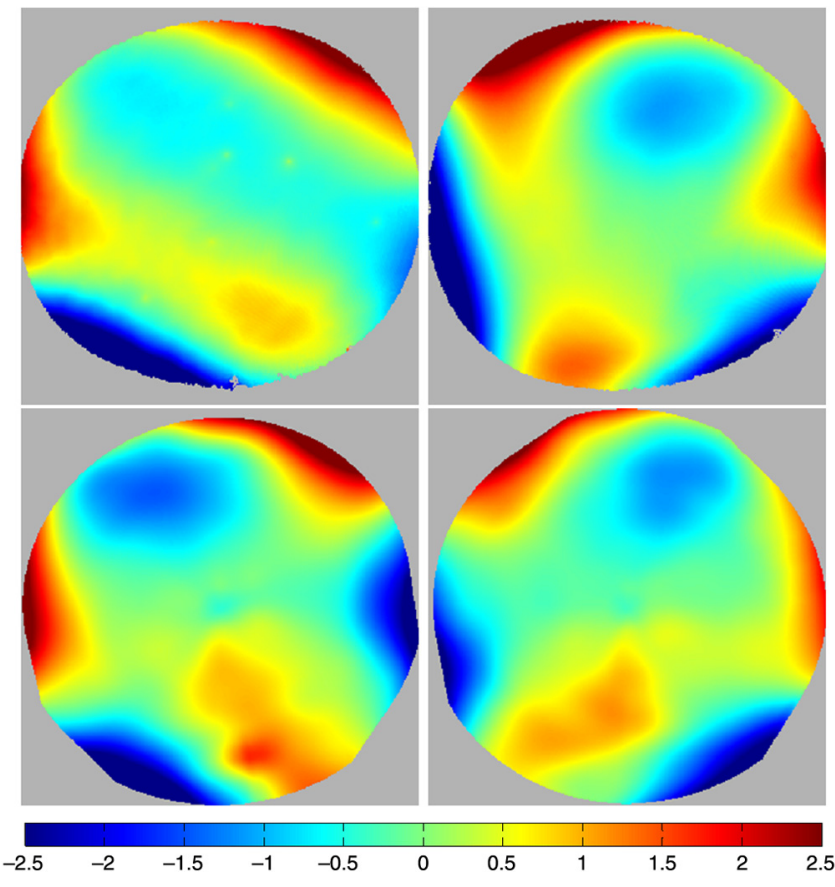

Fig. 24 Subject B, biconic removed corneal surface height (bare eye). Left eye left column, right eye right column, ICT top row, Keratron Piccolo bottom row. Display area is $6 \times 6 \mathrm{~mm} ; \pm 2.5-\mu \mathrm{m}$ color height scale; red is away from eye.

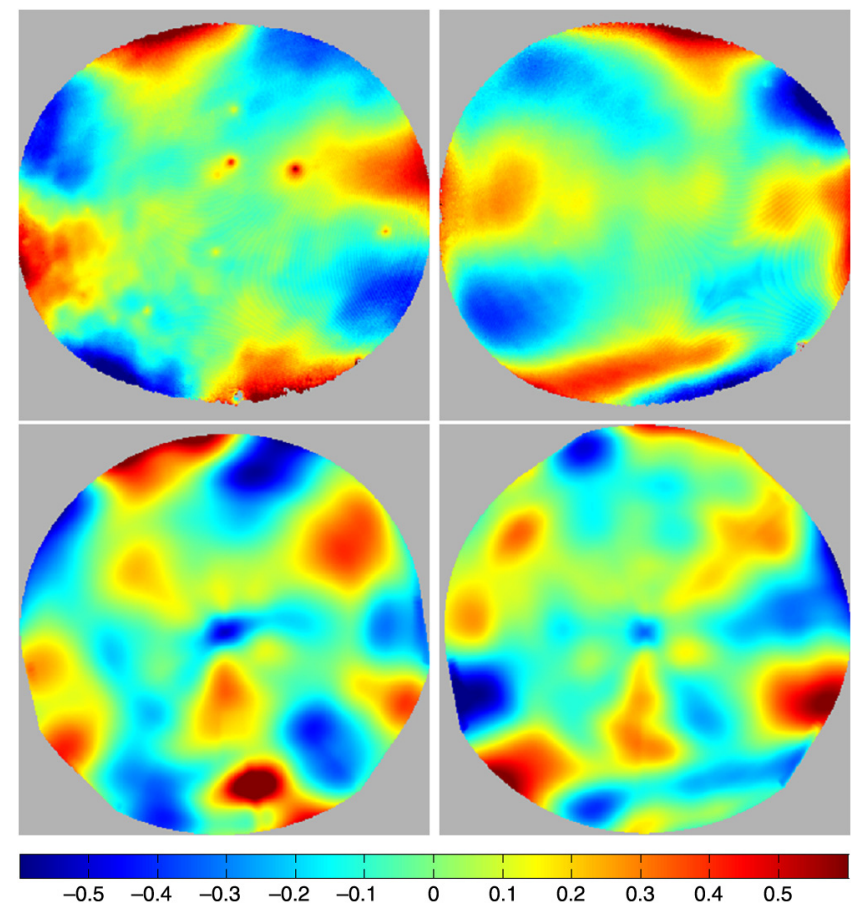

Fig. 25 Subject B, Zernike removed corneal surface height (bare eye). Left eye left column, right eye right column, ICT top row, Keratron Piccolo bottom row. Display area is $6 \times 6 \mathrm{~mm} ; \pm 0.6-\mu \mathrm{m}$ color height scale; red is away from eye. About 15 Zernike terms removed from surface.

resolution of the system $(1.5 \mu \mathrm{m})$, which means that the Keratron Picollo results are effectively noise.

Contact lens. The biconic fit results for Subject B while wearing contact lenses is shown in Table 5. A large portion
Table 5 Subject B, biconic fit results (contact lens).

\begin{tabular}{lcccc} 
& \multicolumn{3}{c}{ Left } & \multicolumn{3}{c}{ Right } \\
\cline { 2 - 5 } Parameters & ICT & Keratron & ICT & Keratron \\
\hline$R x(\mathrm{~mm})$ & -7.899 & -8.120 & -8.076 & -8.113 \\
$R y(\mathrm{~mm})$ & -7.723 & -7.863 & -7.846 & -8.019 \\
$K x$ & -0.283 & -0.222 & -0.231 & -0.401 \\
$K y$ & 0.047 & -0.002 & 0.006 & -0.044 \\
$\theta(\mathrm{deg})$ & -5.40 & -5.06 & 1.90 & 7.82 \\
\hline
\end{tabular}

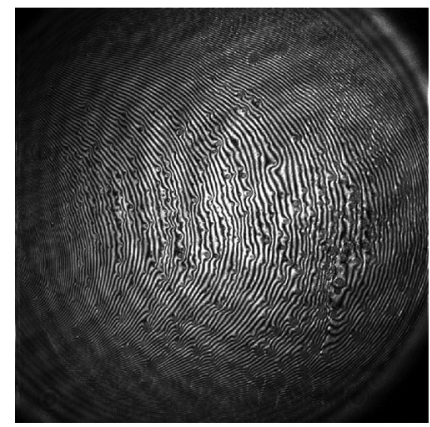

(a)

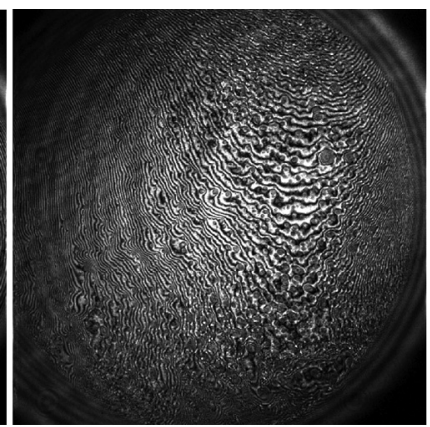

(b)
Fig. 26 Subject B, surface interferograms on eye, wearing contact lenses. (a) Left eye. (b) Right eye.

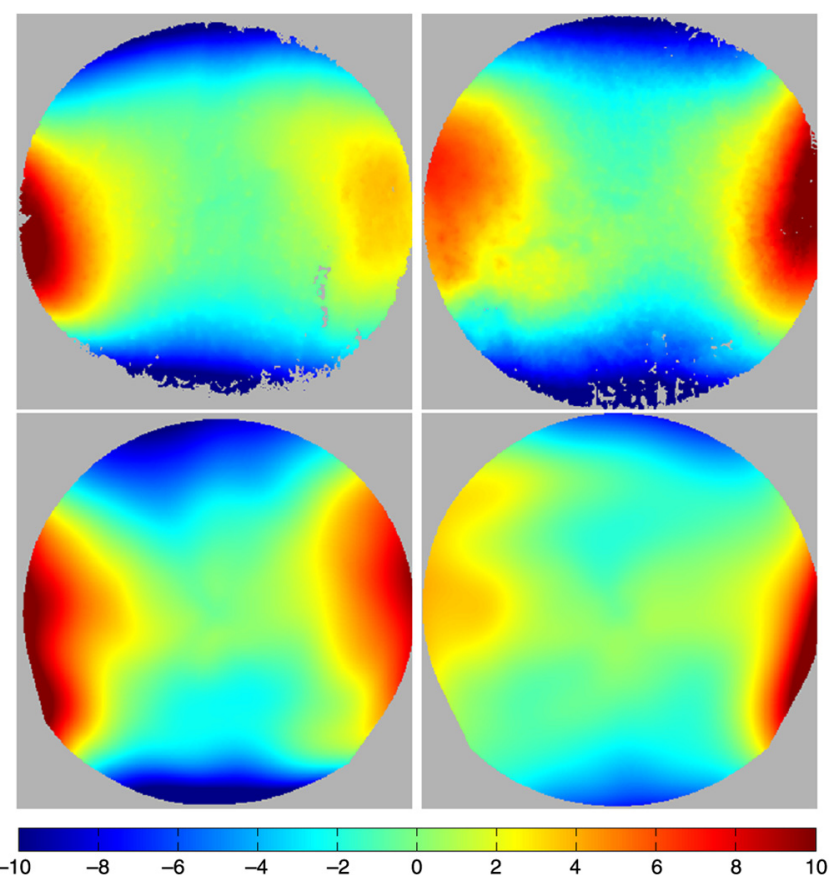

Fig. 27 Subject B, conic removed corneal surface height (contact lens). Left eye left column, right eye right column, ICT top row, Keratron Piccolo bottom row. Display area is $6 \times 6 \mathrm{~mm} ; \pm 10.0-\mu \mathrm{m}$ color height scale; red is away from eye. 

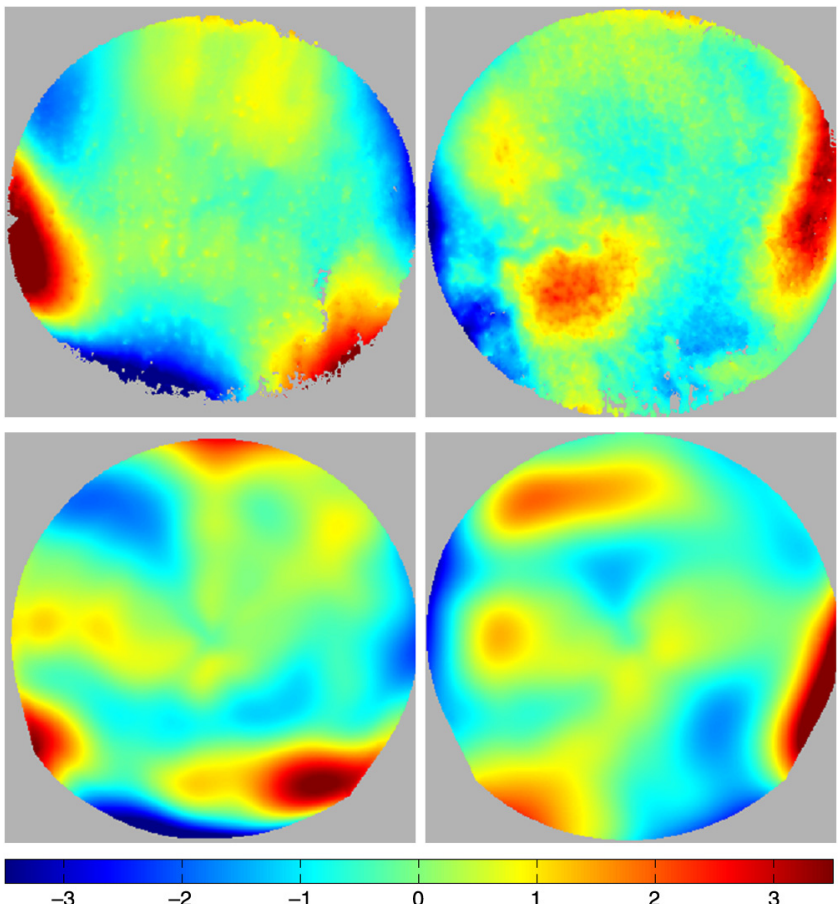

$-3$

$-1$

Fig. 28 Subject $B$, biconic removed corneal surface height (contact lens). Left eye left column, right eye right column, ICT top row, Keratron Piccolo bottom row. Display area is $6 \times 6 \mathrm{~mm} ; \pm 3.5-\mu \mathrm{m}$ color height scale; red is away from eye.

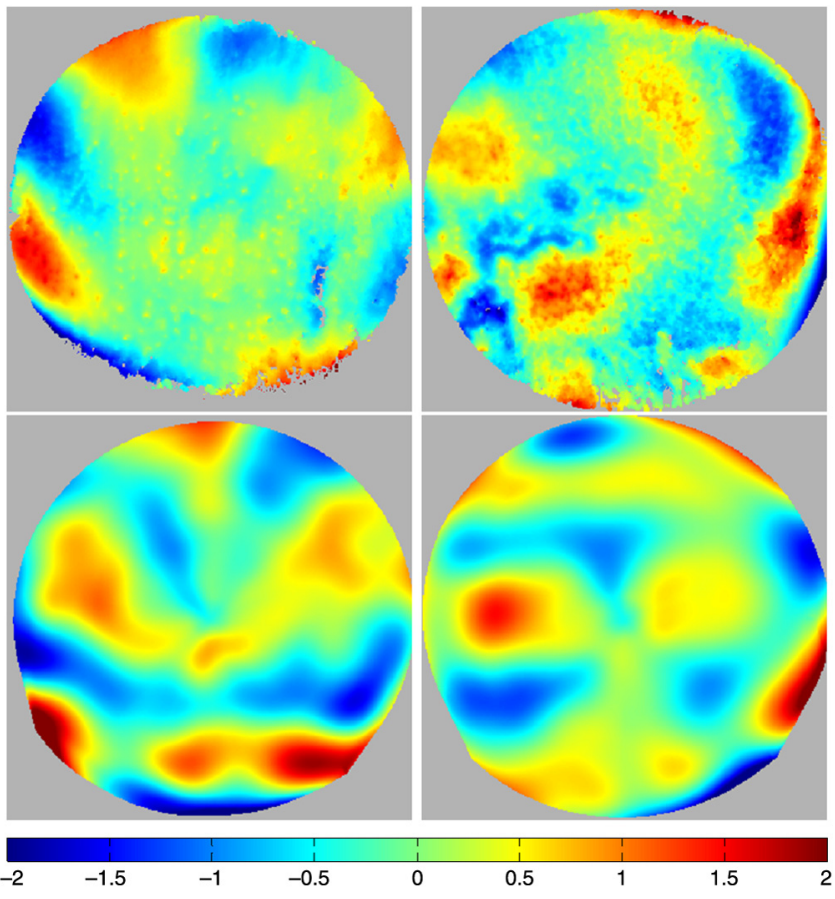

Fig. 29 Subject B, Zernike removed corneal surface height (contact lens). Left eye left column, right eye right column, ICT top row, Keratron Piccolo bottom row. Display area is $6 \times 6 \mathrm{~mm} ; \pm 2-\mu \mathrm{m}$ color height scale; red is away from eye. About 15 Zernike terms removed from surface.

of the subject's corneal astigmatism has been reduced by the toric contact lenses. The remaining astigmatism may be due to the fact that the subject has additional compensating astigmatism from other surfaces in the eye (e.g., the crystalline lens)

such that the contact lenses are not required to fully remove the effects of the corneal astigmatism. Surface interferograms from the ICT are shown in Fig. 26. The residual surface astigmatism can be seen in Fig. 27 with the conic removed surface. The general astigmatic shape appears similar to the bare eye measurement, but the magnitude has been reduced by half. The biconic removed surfaces (Fig. 28) show some of the residual topography, but again, shows more of the mid to high spatialfrequency structure of the tear film. The Zernike removed surfaces (Fig. 29) provide the best display for the higher spatialfrequency structure of the tear film. Some of the residual pentafoil from the bare eye measurement can still be seen in the contact lens measurement. The height scale shows that the Keratron Piccolo is able to resolve surface features; however, the spatial resolution of the system is so large in comparison to the ICT that the complex dynamics that are occurring due to the presence of the tear film are completely lost in the Keratron measurements.

\section{Conclusions}

A dual interferometer system for measuring the dynamic corneal topography and tear film structure has been designed, constructed, demonstrated, and tested on human subjects. This system improves upon the previously reported $\mathrm{TFI}^{62}$ by adding the ability to measure the absolute radius of curvature and corneal topography. On-eye fixation has been implemented to improve testing flexibility and stability.

The ICT has a spatial resolution of $6 \mu \mathrm{m}$, absolute height resolution that is better than $5 \mu \mathrm{m}$, and $25-\mathrm{nm}$ relative height resolution after a best-fit biconic surface is removed. The measurements presented in this paper demonstrate that the ICT has resolution and accuracy that well exceeds that of a commercial Placido disc topographer. High-resolution corneal topography measurements are able to provide feedback on the overall structure of the human cornea. These measurements could be utilized to improve our understanding of vision quality, develop better models for the eye and cornea, or provide feedback on the fitting of contact lenses on the eye. The contact lens measurements demonstrated how important the interaction of the tear film and underlying corneal topography are with the presence of a contact lens. Understanding this interaction could provide feedback for the development of newer contact lens materials or possibly the ability to develop specialized contact lenses that are better matched to the unique topography of individual subjects.

Additionally, the ICT is capable of producing dynamic results-every image that has been displayed in this paper is effectively a single frame out of a 30-FPS movie. These measurements could be displayed sequentially in time to show the dynamics of the absolute corneal topography and tear film. The combination of a noninvasive, high-resolution spatial and height capabilities, absolute topographic reconstruction, and dynamic measurements results in a system that exceeds the capabilities of any other known system.

\section{Acknowledgments}

Funding for this research provided by Johnson \& Johnson Vision Care, Inc.

\section{References}

1. F. J. Holly, "Tear film physiology," Int. Ophthalmol. Clin. 27(1), 2-6 (1987). 
2. D. Benedetto, T. E. Clinch, and P. R. Laibson, "In vivo observation of tear dynamics using fluorophotometry," Arch. Ophthalmol. 102(3), 410-412 (1984).

3. J. Németh et al., "High-speed videotopographic measurement of tear film build-up time," Invest. Ophthalmol. Vis. Sci. 43(6), 1783-1790 (2002).

4. H. T. Kasprzak and T. Licznerski, "Influence of the characteristics of tear film break-up on the point spread function of an eye model," Proc. SPIE 3820, 390-396 (1999).

5. J. P. Guillon, "Tear film structure and contact lenses," in The Preocular Tear Film in Health, Disease and Contact Lens Wear, Vol. 85, pp. 914 939, Dry Eye Institute, Lubbock, Texas (1986).

6. Y. S. Rabinowitz, "Keratoconus," Surv. Ophthalmol. 42(4), 297-319 (1998).

7. L. J. Maguire and W. M. Bourne, "Corneal topography of early keratoconus," Am. J. Ophthalmol. 108(2), 107-112 (1989).

8. M. A. Lemp, "Report of the National Eye Institute/industry workshop on clinical trials in dry eyes," CLAO J. 21, 221-232 (1995).

9. D. A. Schaumberg et al., "Prevalence of dry eye disease among US men: estimates from the Physicians' Health Studies," Arch. Ophthalmol. 127(6), 763-768 (2009).

10. O. D. Schein et al., "Prevalence of dry eye among the elderly," Am. J. Ophthalmol. 124, 723-728 (1997).

11. D. A. Schaumberg et al., "Prevalence of dry eye syndrome among US women," Am. J. Ophthalmol. 136(03), 318-326 (2003).

12. O. D. Schein et al., "Relation between signs and symptoms of dry eye in the elderly. A population-based perspective," Ophthalmology 104, 1395-1401 (1997).

13. L. S. Mengher et al., "A non-invasive instrument for clinical assessment of the pre-corneal tear film stability," Curr. Eye Res. 4(1), 1-7 (1985).

14. T. Kojima et al., "A new noninvasive tear stability analysis system for the assessment of dry eyes," Invest. Ophthalmol. Vis. Sci. 45(5), 13691374 (2004).

15. M. Kopf et al., "Tear film surface quality with soft contact lenses using dynamic videokeratoscopy," J. Optom. 1(1), 14-21 (2008).

16. P. D. Jaycock et al., "Interferometric technique to measure biomechanical changes in the cornea induced by refractive surgery," J. Cataract Refract. Surg. 31(1), 175-184 (2005).

17. J. J. Nichols, "2014 annual report—contact lenses 2014," Contact Lens Spectr. 30, 22-27 (2015).

18. J. J. Nichols and P. E. King-Smith, "Thickness of the pre- and post-contact lens tear film measured in vivo by interferometry," Invest. Ophthalmol. Vis. Sci. 44(1), 68-77 (2003).

19. S. Patel et al., "Effects of fluorescein on tear breakup time and on tear thinning time," Am. J. Optom. Physiol. Opt. 62(3), 188-190 (1985).

20. H. Hamano et al., "Clinical applications of bio differential interference microscope," Eye Contact Lens 6(3), 229-235 (1980).

21. N. Fogt, P. E. King-Smith, and G. Tuell, "Interferometric measurement of tear film thickness by use of spectral oscillations," J. Opt. Soc. Am. A 15(1), 268-275 (1998).

22. P. E. King-Smith et al., "Interferometric imaging of the full thickness of the precorneal tear film," J. Opt. Soc. Am. A 23(9), 2097-2104 (2006).

23. J. I. Prydal and F. W. Campbell, "Study of precorneal tear film thickness and structure by interferometry and confocal microscopy," Invest. Ophthalmol. Vis. Sci. 33(6), 1996-2005 (1992).

24. W. Drexler et al., "Ultrahigh-resolution ophthalmic optical coherence tomography," Nat. Med. 7(4), 502-507 (2001).

25. H. T. Kasprzak, W. Kowalik, and J. Jaroński, "Interferometric measurements of fine corneal topography," Proc. SPIE 2329, 32-39 (1995).

26. T. J. Licznerski, H. T. Kasprzak, and W. Kowalik, "Two interference techniques for in-vivo assessment of the tear film stability on a cornea and contact lens," Proc. SPIE 3320, 183-186 (1998).

27. T. J. Licznerski and H. T. Kasprzak, "Reconstruction of the corneal topography from lateral-shearing interferograms," Proc. SPIE $\mathbf{3 8 2 0}$, 115-119 (1999).

28. T. J. Licznerski, H. T. Kasprzak, and W. Kowalik, "Application of Twyman-Green interferometer for evaluation of in vivo breakup characteristic of the human tear film," J. Biomed. Opt. 4(1), 176-182 (1999).

29. W. Tang et al., "The accuracy and precision performance of four videokeratoscopes in measuring test surfaces," Optom. Vis. Sci. 77(9), 483-491 (2000).
30. D. Priest and R. Munger, "Comparative study of the elevation topography of complex shapes," J. Cataract Refract. Surg. 24(6), 741-750 (1998).

31. M. W. Belin et al., "PAR corneal topography system (PAR CTS): the clinical application of close-range photogrammetry," Optom. Vis. Sci. 72(11), 828-837 (1995).

32. F. H. M. Jongsma et al., "Development of a wide field height eye topographer: validation on models of the anterior eye surface," Optom. Vis. Sci. 75(1), 69-77 (1998).

33. W.-Y. Chang et al., "Heterodyne moiré interferometry for measuring corneal surface profile," Opt. Lasers Eng. 54, 232-235 (2014).

34. L. S. Mengher, K. S. Pandher, and A. J. Bron, "Non-invasive tear film break-up time: sensitivity and specificity," Acta Ophthalmol. 64(4), 441-444 (1986).

35. G. Cairns et al., "Accuracy of orbscan II slit-scanning elevation topography," J. Cataract Refract. Surg. 28(12), 2181-2187 (2002).

36. C. J. Roberts, "Comparison of the EyeSys corneal analysis system and the TMS topographic modeling system using a bicurve test surface," Proc. SPIE 2126, 168-173 (1994).

37. P. M. Kiely, G. Smith, and L. G. Carney, "The mean shape of the human cornea," J. Mod. Opt. 29(8), 1027-1040 (1982).

38. S. Stenstrom, "Investigation of the variation and the correlation of the optical elements of human eyes," Am. J. Optom. Arch. Am. Acad. Optom. 25(8), 218-232 (1948).

39. L. Daily and R. E. Coe, "Lack of effect of anesthetic and mydriatic solutions on the curvature of the cornea," Am. J. Ophthalmol. 53, 49-51 (1962).

40. P. M. Kiely, G. Smith, and L. G. Carney, "Meridional variations of corneal shape," Am. J. Optom. Physiol. Opt. 61(10), 619-626 (1984).

41. A. Guirao and P. Artal, "Corneal wave aberration from videokeratography: accuracy and limitations of the procedure," J. Opt. Soc. Am. A. Opt. Image Sci. Vis. 17(6), 955-965 (2000).

42. J. T. Schwiegerling and J. E. Greivenkamp, "Using corneal height maps and polynomial decomposition to determine corneal aberrations," Optom. Vis. Sci. 74, 906-916 (1997).

43. D. R. Iskander, "Modeling videokeratoscopic height data with spherical harmonics," Optom. Vis. Sci. 86(5), 542-547 (2009).

44. H. T. Kasprzak and D. R. Iskander, "Ultrasonic measurement of fine head movements in a standard ophthalmic headrest," IEEE Trans. Instrum. Meas. 59(1), 164-170 (2010).

45. H. B. Barlow, "Eye movements during fixation," J. Physiol. 116(3), 290-306 (1952).

46. F. Ratliff and L. A. Riggs, "Involuntary motions of the eye during monocular fixation," J. Exp. Psychol. 40(6), 687-701 (1950).

47. R. M. Steinman et al., "Miniature eye movement," Science 181(4102), 810-819 (1973).

48. J. Nachmias, "Determiners of the drift of the eye during monocular fixation," J. Opt. Soc. Am. A 51(7), 761-766 (1961).

49. P. R. Boyce, "The effect of change of target field luminance and colour on fixation eye movements," Opt. Acta 14(3), 213-217 (1967).

50. R. M. Steinman, "Effect of target size, luminance, and color on monocular fixation," J. Opt. Soc. Am. A 55(9), 1158-1165 (1965).

51. J. D. Rattle, "Effect of target size on monocular fixation," Opt. Acta 16(2), 183-190 (1969).

52. R. Engbert and R. Kliegl, "Microsaccades uncover the orientation of covert attention," Vision Res 43(9), 1035-1045 (2003).

53. M. Rolfs, R. Engbert, and R. Kliegl, "Crossmodal coupling of oculomotor control and spatial attention in vision and audition," Exp. Brain Res. 166(3-4), 427-439 (2005).

54. B. J. Tighe, "A decade of silicone hydrogel development: surface properties, mechanical properties, and ocular compatibility," Eye Contact Lens-Sci. Clin. Pract. 39(1), 4-12 (2013).

55. L. C. Thai, A. Tomlinson, and M. G. Doane, "Effect of contact lens materials on tear physiology," Optom. Vision Sci. 81(3), 194-204 (2004).

56. J. E. Greivenkamp et al., "Interferometry and ophthalmics at the College of Optical Sciences," Proc. SPIE 9186, 91860W (2014).

57. G. A. Williby et al., "Interferometric testing of soft contact lenses," Proc. SPIE 5180, 329-339 (2003).

58. E. P. Goodwin, "Dual interferometer system for measuring index of refraction," PhD Disseration, University of Arizona, Tucson, Arizona (2007).

59. K. C. Heideman, "Surface metrology of contact lenses in saline solution," PhD Disseration, University of Arizona, Tucson, Arizona (2014). 
60. B. C. Primeau, J. E. Greivenkamp, and J. J. Sullivan, "In vitro interferometric characterization of dynamic fluid layers on contact lenses," Proc. SPIE 8133, 81330J (2011).

61. B. C. Primeau and J. E. Greivenkamp, "Interferometer for measuring the dynamic surface topography of a human tear film," Proc. SPIE 8215, 821504 (2012).

62. J. D. Micali, J. E. Greivenkamp, and B. C. Primeau, "Dynamic measurement of the corneal tear film with a Twyman-Green interferometer," J. Biomed. Opt. 20(5), 055007 (2015).

63. J. E. Millerd et al., "Pixelated phase-mask dynamic interferometer," Proc. SPIE 5531(520), 304-314 (2004).

64. Z. Malacara and M. Servín, Interferogram Analysis for Optical Testing, 2nd ed., CRC Press, Boca Raton, Florida (2010).

65. C. E. Wieman and L. Hollberg, "Using diode lasers for atomic physics," Rev. Sci. Instrum. 62(1), 1-20 (1991).

66. M. Takeda, "Spatial-carrier fringe-pattern analysis and its applications to precision interferometry and profilometry: an overview," Ind. Metrol. 1(2), 79-99 (1990).

67. M. Takeda, H. Ina, and S. Kobayashi, "Fourier-transform method of fringe-pattern analysis for computer-based topography and interferometry," J. Opt. Soc. Am. A 72(1), 156-160 (1982).

68. L. Thaler et al., "What is the best fixation target? The effect of target shape on stability of fixational eye movements," Vision Res. 76, 31-42 (2013).

69. B. C. Primeau, G. L. Goldstein, and J. E. Greivenkamp, "Laser exposure analysis for a near- infrared ocular interferometer," Opt. Eng. 51(6), 064301 (2012).

70. ANSI, American National Standard for Safe Use of Lasers, ANSI Z136.1-2007 American National Standards Institute, New York (2007).

71. D. C. Ghiglia and M. D. Pritt, Two-Dimensional Phase Unwrapping: Theory, Algorithms, and Software, Wiley, New York(1998).

72. R. J. Braun et al., "Dynamics and function of the tear film in relation to the blink cycle," Prog. Retin. Eye Res. 45, 132-164 (2015).

73. J. D. Micali, "Interferometer for measuring dynamic corneal topography," PhD Disseration, University of Arizona, Tucson, Arizona (2015).
74. L. A. Selberg, "Interferometer accuracy and precision," Proc. SPIE 1400, 24-32 (1991).

75. A. E. Lowman and J. E. Greivenkamp, "Interferometer induced wavefront errors when testing in a non-null configuration," Proc. SPIE 2004, 173-181 (1994).

76. J. M. Geary, M. Yoo, and G. Si, "Retrace error: a case study," Proc. SPIE 1776, 98-105 (1992).

77. J. D. Micali and J. E. Greivenkamp, "Method for reconstruction of complex surface shapes from a reflection-based non-null interferometric measurement," Opt. Eng. 55(3), 034101 (2016).

78. Z. Malacara and D. Malacara, "Design of lenses to project the image of a pupil in optical testing interferometers," Appl. Opt. 34(4), 739-742 (1995).

79. R. O. Gappinger and J. E. Greivenkamp, "Iterative reverse optimization procedure for calibration of aspheric wave-front measurements on a nonnull interferometer," Appl. Opt. 43(27), 5152-5161 (2004).

80. H. J. Jeong and G. N. Lawrence, "Simultaneous determination of misalignment and mirror surface figure error of a three mirror off-axis telescope by end-to-end measurements and reverse optimization: numerical analysis and simulation," Proc. SPIE 966, 341-353 (1988).

81. I. Powell, "Employment of reverse optimization to relax manufacturing tolerances imposed on system constructional parameters associated with complex optical systems," Appl. Opt. 39(13), 2174-2183 (2000).

82. C. Roberts, "Characterization of the inherent error in a sphericallybiased corneal topography system in mapping a radially aspheric surface," J. Refract. Corneal Surg. 10(2), 103-111 (1994).

Jason D. Micali received his MS and PhD degrees in optical sciences from the College of Optical Sciences, University of Arizona, in 2012 and 2015, respectively. His research interests include electro-optical systems, interferometry and optical testing, and metrology.

John E. Greivenkamp is a professor at the College of Optical Sciences, University of Arizona. He has served as a member of the SPIE board of directors and also the editor of the SPIE Field Guide Series. He is the author of SPIE Field Guide to Geometrical Optics. 